MATHEMATICS OF COMPUTATION

Volume 80, Number 274, April 2011, Pages 625-648

S $0025-5718(2010) 02435-4$

Article electronically published on November 16, 2010

\title{
AFEM FOR THE LAPLACE-BELTRAMI OPERATOR ON GRAPHS: DESIGN AND CONDITIONAL CONTRACTION PROPERTY
}

\author{
KHAMRON MEKCHAY, PEDRO MORIN, AND RICARDO H. NOCHETTO
}

\begin{abstract}
We present an adaptive finite element method (AFEM) of any polynomial degree for the Laplace-Beltrami operator on $C^{1}$ graphs $\Gamma$ in $\mathbb{R}^{d}(d \geq$ 2 ). We first derive residual-type a posteriori error estimates that account for the interaction of both the energy error in $H^{1}(\Gamma)$ and the surface error in $W_{\infty}^{1}(\Gamma)$ due to approximation of $\Gamma$. We devise a marking strategy to reduce the total error estimator, namely a suitably scaled sum of the energy, geometric, and inconsistency error estimators. We prove a conditional contraction property for the sum of the energy error and the total estimator; the conditional statement encodes resolution of $\Gamma$ in $W_{\infty}^{1}$. We conclude with one numerical experiment that illustrates the theory.
\end{abstract}

\section{INTRODUCTION}

We consider a surface $\Gamma \subset \mathbb{R}^{d}$ described as the graph of a function $z(\mathbf{x})$ defined on a bounded polygonal/polyhedral region $\Omega \subset \mathbb{R}^{d-1}, d \geq 2$, i.e.,

$$
\Gamma:=\left\{(\mathbf{x}, z(\mathbf{x})) \in \mathbb{R}^{d} \mid \mathbf{x} \in \Omega \subset \mathbb{R}^{d-1}\right\},
$$

where $z: \Omega \rightarrow \mathbb{R}$ is a $C^{1}$ function. In contrast to the works by Demlow and Dziuk 13, 10, 9, we do not need $C^{2}$ regularity. In fact, we may even allow $z$ to be Lipschitz with discontinuities of $\nabla z$ in $\Omega$ aligned with the initial mesh. We will not dwell on this matter but instead refer to \$5 where we allow $z$ to be piecewise smooth.

We consider the Dirichlet boundary value problem for the Laplace-Beltrami operator $\Delta_{\Gamma}$ on $\Gamma$

$$
-\Delta_{\Gamma} u=f \text { on } \Gamma, \quad u=0 \text { on } \partial \Gamma,
$$

with $f \in L^{2}(\Gamma)$. For simplicity we consider homogeneous Dirichlet data, but a non-zero Dirichlet or Neumann boundary condition can be treated as well; see [18].

Received by the editor July 20, 2009 and, in revised form, February 26, 2010.

2010 Mathematics Subject Classification. Primary 65N30, 65N50.

Key words and phrases. Laplace-Beltrami operator, graphs, adaptive finite element method, a posteriori error estimate, energy and geometric errors, bisection, contraction.

The first author was partially supported by NSF Grants DMS-0204670, DMS-0505454, and INT-0126272.

The second author was partially supported by CONICET through Grants PIP 5478, PIP 112200801-02182, by Universidad Nacional del Litoral through Grants CAI+D 008-054 and CAI+D PI 062-312, and by NSF Grant DMS-0204670.

The third author was partially supported by NSF Grants DMS-0204670, DMS-0505454, DMS0807811, and INT-0126272, and the General Research Board of the University of Maryland.

(C)2010 American Mathematical Society 
Elliptic partial differential equations (PDE) on surfaces are ubiquitous from geometry and relativity theory to applications in phase transitions, materials science, and image processing. They are typically governed by the Laplace-Beltrami operator $\Delta_{\Gamma}$, but more general operators arise as well. We refer to Dziuk 13 for the formulation of FEM for the Laplace-Beltrami operator on parametric surfaces and corresponding a priori error analysis, as well as to Demlow and Dziuk [10 for a posteriori error analysis, both for linear finite elements; we also mention Demlow [9] which extends the a priori error analysis of [13] to any polynomial degree and the $L^{\infty}$-norm. In contrast to [10], our approach does not use a level set function (such as $x_{d}-z(\mathbf{x})=0$ ) nor the distance function to represent $\Gamma$, and is valid for any polynomial degree. In this vein, this paper presents a constructive and flexible building block that extends to parametric surfaces without requiring the distance function [5]. As in [10], we show a posteriori error estimates that consist of two parts: the energy and geometric error estimators; this is a dual version of the a priori error estimates in [13. However, as opposed to [10, 13], our geometric error estimator is of the same order as the energy error estimator because we cannot exploit special orthogonality properties associated to the distance function. This is the price to pay for flexibility in describing the surface.

In addition, we design an AFEM, prove reduction properties for the estimator, geometric error and surface estimator, and use them to show a conditional contraction property of AFEM. The latter appears to be the first result for the Laplace-Beltrami operator.

We next introduce the weak formulation, the FEM, and give an outline of the paper along with our main result.

1.1. Variational formulation. Given a function $v: \Gamma \rightarrow \mathbb{R}$ we define its lift $\tilde{v}: \Omega \times \mathbb{R} \rightarrow \mathbb{R}$ as the obvious extension

$$
\tilde{v}\left(\mathbf{x}, x_{d}\right):=v(\mathbf{x}, z(\mathbf{x})), \quad \forall \mathbf{x} \in \Omega, x_{d} \in \mathbb{R} .
$$

We denote by $\nabla \tilde{v} \in \mathbb{R}^{d}$ the gradient of $\tilde{v}$, arranged as a row vector, by $q$ the elementary surface area, and by $\nu$ the unit normal vector to $\Gamma$ again arranged as a row vector, namely,

$$
q:=\sqrt{1+|\nabla z|^{2}}, \quad \nu:=\frac{1}{q}(-\nabla z, 1) .
$$

We indicate with $\nabla_{\Gamma} v \in \mathbb{R}^{d}$ the tangential gradient of $v$ on $\Gamma$, which is the component of $\nabla \tilde{v}$ tangent to $\Gamma$ :

$$
\nabla_{\Gamma} v=\nabla \tilde{v}-(\nabla \tilde{v} \cdot \nu) \nu=\nabla \tilde{v}\left(\mathbf{I}-\nu^{T} \nu\right) .
$$

Likewise, the tangential divergence of a vector field $\mathbf{q}: \Omega \times \mathbb{R} \rightarrow \mathbb{R}^{d}$ is given by

$$
\operatorname{div}_{\Gamma} \mathbf{q}=\operatorname{div} \mathbf{q}-\nu D \mathbf{q} \nu^{T}
$$

where $D \mathbf{q}$ stands for the differential matrix of $\mathbf{q}$; hence, we have

$$
\Delta_{\Gamma} v=\operatorname{div}_{\Gamma} \nabla_{\Gamma} v=\Delta \tilde{v}-(\nabla \tilde{v} \cdot \nu)(\operatorname{div} \nu)-\nu D^{2} \tilde{v} \nu^{T}
$$

where $D^{2} \tilde{v} \in \mathbb{R}^{d \times d}$ is the Hessian of $\tilde{v}$ and $\operatorname{div} \nu=\operatorname{div}_{\mathbf{x}}\left(\frac{\nabla z}{q}\right)$ is the mean curvature of $\Gamma$. 
To formulate (1.1) weakly, we now introduce Sobolev spaces on the surface $\Gamma$ :

$$
\begin{aligned}
& H^{1}(\Gamma):=\left\{v \in L^{2}(\Gamma) \mid \nabla_{\Gamma} v \in L^{2}(\Gamma)\right\}, \\
& H_{0}^{1}(\Gamma):=\left\{v \in H^{1}(\Gamma) \mid v \text { has zero trace on } \partial \Gamma\right\} .
\end{aligned}
$$

The weak formulation of (1.1) is thus

$$
u \in H_{0}^{1}(\Gamma): \quad \int_{\Gamma} \nabla_{\Gamma} u \nabla_{\Gamma}^{T} v=\int_{\Gamma} f v \quad \forall v \in H_{0}^{1}(\Gamma) .
$$

This problem admits a unique solution due to the Lax-Milgram lemma and the fact that $L^{2}(\Gamma)$ belongs to the topological dual space of $H_{0}^{1}(\Gamma)$.

1.2. The finite element method on graphs. To approximate (1.7) via the FEM we proceed as follows. Given an initial conforming partition $\mathcal{T}_{0}(\Omega)$ of $\Omega$ into simplices, we consider a sequence of conforming nested refinements $\mathcal{T}_{k}(\Omega)$ into shape regular simplices using bisection. Next, we let $\mathbb{V}_{k}(\Omega)$ be the finite element space over $\mathcal{T}_{k}(\Omega)$ consisting of $C^{0}$ piecewise polynomial functions of degree $n \geq 1$; this yields $\mathbb{V}_{k}(\Omega) \subset \mathbb{V}_{k+1}(\Omega)$ for all $k$. We let $z_{k} \in \mathbb{V}_{k}(\Omega)$ be the Lagrange interpolant of $z$ in $\mathbb{V}_{k}(\Omega)$; the image $\Gamma_{k}:=\left\{\left(\mathbf{x}, z_{k}(\mathbf{x})\right): \mathbf{x} \in \Omega\right\}$ is thus a piecewise polynomial approximation to $\Gamma$. This induces a one-to-one correspondence between elements $\widehat{T} \in \mathcal{T}_{k}(\Omega)$ and elements $T \in \mathcal{T}_{k}\left(\Gamma_{k}\right), \widetilde{T} \in \mathcal{T}_{k}(\Gamma)$ defined by

$$
T=\left\{\left(\mathbf{x}, z_{k}(\mathbf{x})\right): \mathbf{x} \in \widehat{T}\right\}, \quad \widetilde{T}:=\{(\mathbf{x}, z(\mathbf{x})): \mathbf{x} \in \widehat{T}\} .
$$

The space $\mathbb{V}_{k}\left(\Gamma_{k}\right)$ is simply the lift of $\mathbb{V}_{k}(\Omega)$ via (1.2) where $z$ is replaced by $z_{k}$, and $\stackrel{\circ}{\mathbb{V}}_{k}\left(\Gamma_{k}\right):=\mathbb{V}_{k}\left(\Gamma_{k}\right) \cap H_{0}^{1}\left(\Gamma_{k}\right)$. Note, however, that $\mathbb{V}_{k}\left(\Gamma_{k}\right)$ is no longer a subspace of $\mathbb{V}_{k+1}\left(\Gamma_{k+1}\right)$. This lack of consistency must be accounted for in the error analysis.

We are now ready to introduce the FEM for the Laplace-Beltrami operator on graphs. If $F_{k} \in L^{2}\left(\Gamma_{k}\right)$ is a suitable approximation of $f$, then the finite element function $U_{k}: \Gamma_{k} \rightarrow \mathbb{R}$ solves

$$
U_{k} \in \stackrel{\circ}{\mathbb{V}}_{k}: \quad \int_{\Gamma_{k}} \nabla_{\Gamma_{k}} U_{k} \nabla_{\Gamma_{k}}^{T} V=\int_{\Gamma_{k}} F_{k} V \quad \forall V \in \stackrel{\circ}{\mathbb{V}}_{k} .
$$

This yields a symmetric positive definite (SPD) linear system which can be solved with standard linear algebra tools. We note that (1.9) can be thought of as a linear elliptic PDE with variable coefficients in $\Omega$; see Remark 2.1, However, in view of implementation issues and generalizations to parametric surfaces, carried out in [5], we prefer to think of (1.9) as defined on $\Gamma_{k}$.

1.3. Main result and outline. The main purpose of this paper is to design an AFEM for (1.1), prove a contraction property and thus convergence of AFEM, and document performance of AFEM computationally. We now briefly state our main result and provide an outline of the paper.

Let $\left(\mathbb{V}_{k}\left(\Gamma_{k}\right), \mathcal{T}_{k}\left(\Gamma_{k}\right)\right)_{k=1}^{\infty}$ be a sequence of space-mesh pairs created via the following loop

$$
\text { SOLVE } \rightarrow \text { ESTIMATE } \rightarrow \text { MARK } \rightarrow \text { REFINE }
$$

as described below in 3 . We start with the usual interior and jump residual indicators

$$
\begin{aligned}
\left.\mathcal{R}\left(U_{k}\right)\right|_{T} & :=\left.\left(F_{k}+\Delta_{\Gamma_{k}} U_{k}\right)\right|_{T} \quad \forall T \in \mathcal{T}_{k}\left(\Gamma_{k}\right), \\
\left.\mathcal{J}\left(U_{k}\right)\right|_{S} & :=\left.\left(\nabla_{\Gamma_{k}} U_{k}\right)\right|_{T^{+}} \cdot \mathbf{n}_{S}^{+}+\left.\left(\nabla_{\Gamma_{k}} U_{k}\right)\right|_{T^{-}} \cdot \mathbf{n}_{S}^{-} \quad \forall S \in \mathcal{S}_{k}^{o},
\end{aligned}
$$


where $\mathcal{S}_{k}^{o}$ denotes the set of interior sides of $\mathcal{T}_{k}\left(\Gamma_{k}\right)$, and $\left.\mathcal{J}\left(U_{k}\right)\right|_{S}=0$ if $S$ is a boundary side. Given $S \in \mathcal{S}_{k}^{o}, \mathbf{n}_{S}^{+}$and $\mathbf{n}_{S}^{-}$denote the outward unit normals to $S$ with respect to the adjacent elements $T^{+}, T^{-} \in \mathcal{T}_{k}\left(\Gamma_{k}\right)$ sharing $S$ and tangent to them, respectively. For any polynomial degree $n \geq 1, \mathbf{n}_{S}^{+}\left(\operatorname{resp} . \mathbf{n}_{S}^{-}\right)$results from lifting, via the map (2.1) below, the unit normal vector to $\widehat{T}^{+}$(resp. $\widehat{T}^{-}$) to the tangent plane to $T^{+}$(resp. $T^{-}$) at the corresponding point of $S$, followed by normalization to unit size. Therefore, for $n=1, \mathbf{n}_{S}^{+}\left(\operatorname{resp} . \mathbf{n}_{S}^{-}\right)$is a constant vector along $S$, but this is not the case for $n>1$.

We define the usual energy error indicator $\eta_{k}(T)$ by [1, 21]

$$
\eta_{k}^{2}(T):=h_{T}^{2}\left\|\mathcal{R}\left(U_{k}\right)\right\|_{L^{2}(\widehat{T})}^{2}+h_{T}\left\|\mathcal{J}\left(U_{k}\right)\right\|_{L^{2}(\partial \widehat{T})}^{2} \quad \forall T \in \mathcal{T}_{k}\left(\Gamma_{k}\right)
$$

where $h_{T}:=|\widehat{T}|^{\frac{1}{d-1}}$ is the meshsize of $T$. Since the integrals are computed over $\widehat{T}$ and $\partial \widehat{T}$, which are contained in $\Omega$, we commit a slight abuse of notation in writing the integrands as $\mathcal{R}\left(U_{k}\right), \mathcal{J}\left(U_{k}\right)$ instead of the corresponding lifts $\widetilde{\mathcal{R}\left(U_{k}\right)}, \widetilde{\mathcal{J}\left(U_{k}\right)}$ given by (1.2). Note that $b$ bisections of $T$ reduce its meshsize by a factor $2^{-\frac{b}{d-1}}$. We then let the energy error estimator be $\eta_{k}:=\left(\sum_{T \in \mathcal{T}_{k}\left(\Gamma_{k}\right)} \eta_{k}^{2}(T)\right)^{1 / 2}$.

A key novelty for the Laplace-Beltrami operator is the interaction between geometry and PDE encoded in (1.15) below. To quantify the approximation of $\Gamma$ by $\Gamma_{k}$ in $W_{\infty}^{1}$, we let the surface estimator be $\lambda_{k}:=\max _{T \in \mathcal{T}_{k}\left(\Gamma_{k}\right)} \lambda_{k}(T)$, where

$$
\lambda_{k}(T):=\left\|\nu-\nu_{k}\right\|_{L^{\infty}(\widehat{T})} \quad \forall T \in \mathcal{T}_{k}\left(\Gamma_{k}\right),
$$

and the unit normals $\nu$ and $\nu_{k}$ to $\Gamma$ and $\Gamma_{k}$ are defined according to (1.3); note that $\lambda_{k}$ accumulates in $\ell^{\infty}\left(\mathcal{T}_{k}\left(\Gamma_{k}\right)\right)$. Next, we introduce the geometric estimator $\zeta_{k}:=\left(\sum_{T \in \mathcal{T}_{k}\left(\Gamma_{k}\right)} \zeta_{k}^{2}(T)\right)^{1 / 2}$, where

$$
\zeta_{k}(T):=\lambda_{k}(T)\left\|\nabla_{\Gamma} U_{k}\right\|_{L^{2}(\widetilde{T})} \quad \forall T \in \mathcal{T}_{k}\left(\Gamma_{k}\right),
$$

and observe that its accumulation is in $\ell^{2}\left(\mathcal{T}_{k}\left(\Gamma_{k}\right)\right)$. Moreover, we point out that the second factor in (1.15) is evaluated on $\Gamma$. Similarly, we define the energy error to be

$$
e_{k}:=\left\|\nabla_{\Gamma}\left(u-U_{k}\right)\right\|_{L^{2}(\Gamma)},
$$

where the integral is on $\Gamma$ and so employs $\nabla_{\Gamma}$ along with the lift (1.2) for $U_{k}$. Finally, to quantify the lack of consistency of the right-hand side with the discrete surface, we define the local quantity

$$
\rho_{k}^{2}(T):=\lambda_{k}^{2}(T) h_{T}^{2}\|f\|_{L^{2}(T)}^{2} \quad \forall T \in \mathcal{T}_{k},
$$

and the corresponding inconsistency estimator $\rho_{k}^{2}:=\sum_{T \in \mathcal{T}_{k}} \rho_{k}^{2}(T)$.

We are now ready to state the main result of this paper, the conditional contraction property between consecutive iterates of the adaptive loop (1.10) proved in 幽 
Let $\left(\Gamma_{0}, \mathcal{T}_{0}\right)$ be an arbitrary initial surface-mesh pair of $\Gamma$. There exist positive constants $\gamma_{0}, \beta_{1}, \beta_{2}, \Lambda$, and $\alpha<1$, solely depending on $\left(\Gamma_{0}, \mathcal{T}_{0}\right)$, shape regularity and the user's parameters of AFEM, such that if the total estimator employed by AFEM is given by

$$
\mathcal{E}_{k}^{2}:=\eta_{k}^{2}+\beta_{1} \zeta_{k}^{2}+\beta_{2} \rho_{k}^{2},
$$

and $\lambda_{k} \leq \Lambda$, then

$$
e_{k+1}^{2}+\gamma_{0} \mathcal{E}_{k+1}^{2} \leq \alpha\left(e_{k}^{2}+\gamma_{0} \mathcal{E}_{k}^{2}\right)
$$

Note that the condition $\lambda_{k} \leq \Lambda$ encodes suitable resolution of $\Gamma$ for AFEM to be contractive. This result extends to the Laplace-Beltrami operator on graphs, the contraction property for symmetric elliptic PDE recently proved by Cascón et al. 4, but a few new geometric ingredients are added.

The rest of the paper is organized as follows. We start in $\$ 2$ with a review of differential geometry on graphs. We discuss the procedures SOLVE, ESTIMATE, MARK and REFINE, along with AFEM in $\$ 3$. We prove several basic properties of AFEM in $\$ 4.1$ and its contraction property in $\$ 4.2$. We conclude in $\$ 5$ with a numerical experiment that sheds light on the theory and documents the performance of AFEM on graphs.

To avoid confusion with constants, we write $a \lesssim b$ to denote $a \leq C b$ for some constant $C$ depending only on shape regularity of the mesh $\mathcal{T}_{k}(\Omega)$ and the Lipschitz regularity of the surface $\Gamma$ through $\|\nabla z\|_{L^{\infty}(\Omega)}$; we also write $a \approx b$ to indicate $b \lesssim a \lesssim b$. We use the notation $e_{k}(\omega), \eta_{k}(\omega), \lambda_{k}(\omega), \zeta_{k}(\omega)$ and $\rho_{k}(\omega)$ to indicate

restrictions of these quantities to a subset $\omega$ of $\Gamma_{k}$. Finally, we abbreviate $\mathbb{V}_{k}\left(\Gamma_{k}\right)$ and $\mathcal{T}_{k}\left(\Gamma_{k}\right)$ with $\stackrel{\circ}{V}_{k}$ and $\mathcal{T}_{k}$.

\section{Differential GeOMetry ON GRAPHS}

According to the lifting (1.2), a function $v: \Gamma \rightarrow \mathbb{R}$ induces a function $\hat{v}: \Omega \rightarrow \mathbb{R}$ upon setting $\hat{v}(\mathbf{x})=v(\mathbf{x}, z(\mathbf{x}))=\tilde{v}\left(\mathbf{x}, x_{d}\right)$. Therefore, $\nabla \tilde{v}=\left(\nabla_{\mathbf{x}} \hat{v}, 0\right)$ and (1.4) becomes

$$
\nabla_{\Gamma} v=\nabla \tilde{v} \tilde{\mathbf{D}}=\nabla_{\mathbf{x}} \hat{v} \mathbf{D},
$$

where $\tilde{\mathbf{D}} \in \mathbb{R}^{d \times d}$ and $\mathbf{D} \in \mathbb{R}^{(d-1) \times d}$ are the matrices

$$
\tilde{\mathbf{D}}:=\mathbf{I}_{d \times d}-\nu^{T} \nu, \quad \mathbf{D}=\left[\mathbf{I}_{(d-1) \times(d-1)} \mathbf{0}\right]+q^{-2} \nabla z^{T}(-\nabla z, 1) ;
$$

we see that $\mathbf{D}$ results from $\tilde{\mathbf{D}}$ upon eliminating its last row and their 2-norms satisfy $|\mathbf{D}|=|\tilde{\mathbf{D}}|=1$. When no confusion is possible, we will refer to the three functions $v, \tilde{v}, \hat{v}$ just as $v$. In view of (2.1), we can express (1.7) as an elliptic PDE with variable coefficients in $\Omega$ :

$$
\int_{\Gamma} \nabla_{\Gamma} u \nabla_{\Gamma}^{T} \varphi=\int_{\Omega} \nabla_{\mathbf{x}} u\left(q \mathbf{D} \mathbf{D}^{T}\right) \nabla_{\mathbf{x}}^{T} \varphi .
$$

Moreover, a simple calculation shows that $\mathbf{D D}^{T}$ is SPD and has the form

$$
\mathbf{D D}^{T}=\mathbf{I}_{(d-1) \times(d-1)}-q^{-2}\left(\nabla z^{T} \nabla z\right) .
$$


To write a sort of inverse formula to (2.1), we let $\mathbf{t}_{i}(\mathbf{x})=\left(\mathbf{e}_{i}, \frac{\partial z(\mathbf{x})}{\partial x_{i}}\right)^{T} \in \mathbb{R}^{d}$ be the tangent (column) vectors to $\Gamma$ with $\left\{\mathbf{e}_{i}\right\}_{i=1}^{d-1}$ the canonical basis of $\mathbb{R}^{d-1}$. If

$$
\mathbf{T}(\mathbf{x}):=\left[\mathbf{t}_{1}(\mathbf{x}), \mathbf{t}_{2}(\mathbf{x}), \ldots, \mathbf{t}_{d-1}(\mathbf{x})\right]=\left[\begin{array}{c}
\mathbf{I}_{(d-1) \times(d-1)} \\
\nabla z
\end{array}\right] \in \mathbb{R}^{d \times(d-1)},
$$

then $|\mathbf{T}(\mathbf{x})|=q(\mathbf{x})$ and the chain rule applied to $\hat{v}(\mathbf{x})=\tilde{v}(\mathbf{x}, z(\mathbf{x}))$ yields the relations

$$
\nabla_{\mathbf{x}} \hat{v}(\mathbf{x})=\nabla v(\mathbf{x}, z(\mathbf{x})) \mathbf{T}(\mathbf{x})=\nabla_{\Gamma} v(\mathbf{x}, z(\mathbf{x})) \mathbf{T}(\mathbf{x}) \quad \forall \mathbf{x} \in \Omega .
$$

It is easy to verify by simple matrix multiplication that $\mathbf{D}$ and $\mathbf{T}$ are pseudoinverses, namely,

$$
\mathbf{D T}=\mathbf{I}_{(d-1) \times(d-1)}, \quad \mathbf{T D}=\mathbf{I}_{d \times d}-\nu^{T} \nu ;
$$

note that $(\mathbf{T D})^{2}=\mathbf{T} \mathbf{D} \mathbf{D}^{T} \mathbf{T}^{T}=\mathbf{T D}$. Similar results also apply elementwise to $z_{k}$ in place of $z$, that is, $\mathbf{D}_{k}, \mathbf{T}_{k}$, and $q_{k}$ are defined for each $T \in \mathcal{T}_{k}(\Omega)$ using $z_{k}$ instead of $z$, and the same equalities (2.5) and (2.6) hold for them.

Remark 2.1 (PDE with variable coefficients). If $\mathbf{A}:=q \mathbf{D} \mathbf{D}^{T}$ and $F:=q f$, then (1.7) becomes

$$
-\operatorname{div}\left(\mathbf{A} \nabla^{T} u\right)=F \quad \text { in } \quad \Omega, \quad u=0 \quad \text { on } \quad \partial \Omega .
$$

Convergence of AFEM for this type of PDE with variable coefficients $\mathbf{A}$ is studied by Mekchay and Nochetto [15, who in turn exploit an idea of Chen and Feng [6]; see also Morin, Nochetto and Siebert [16, 17] for piecewise constant A. A quasioptimal convergence rate is derived by Cascón et al. 4. However, we prefer to view the surface $\Gamma$ as a geometric object to be discretized, and the PDE to be formulated directly on $\Gamma$ and $\Gamma_{k}$. This is consistent with the a priori analysis of Dziuk [13] (see also [9]), and extends naturally to parametric surfaces [10, 5].

Remark 2.2 (Quadrature). We could regard the approximation of $\Gamma$ by $\Gamma_{k}$ as quadrature in that the coefficient matrix $\mathbf{A}$ of Remark 2.1 is replaced by $\mathbf{A}_{k}:=q_{k} \mathbf{D}_{k} \mathbf{D}_{k}^{T}$. This is not, however, interpolatory quadrature because $\mathbf{A}$ is not evaluated at preassigned points: the value of $\mathbf{A}_{k}$ within an element depends on all the values of $z(\mathbf{x})$ at its nodes. To get intuition about the structure of the error committed in replacing $\mathbf{A}$ by $\mathbf{A}_{k}$, let $u_{k} \in H_{0}^{1}(\Omega)$ be the solution of the PDE

$$
-\operatorname{div}\left(\mathbf{A}_{k} \nabla^{T} u_{k}\right)=F, \quad \text { in } \Omega .
$$

The error $e=u-u_{k} \in H_{0}^{1}(\Omega)$ thus satisfies for all $v \in H_{0}^{1}(\Omega)$,

$$
\int_{\Omega} \nabla e \mathbf{A} \nabla^{T} v=\int_{\Omega} \nabla u_{k}\left(\mathbf{A}_{k}-\mathbf{A}\right) \nabla^{T} v=\int_{\Omega} \nabla u_{k}\left(\mathbf{A}_{k}-\mathbf{A}\right) \mathbf{A}^{-\frac{1}{2}} \mathbf{A}^{\frac{1}{2}} \nabla^{T} v,
$$

whence for $v=e$,

$$
\int_{\Omega} \nabla e \mathbf{A} \nabla^{T} e \leq \int_{\Omega} \nabla u_{k}\left(\left(\mathbf{A}_{k}-\mathbf{A}\right) \mathbf{A}^{-1}\left(\mathbf{A}_{k}-\mathbf{A}\right)\right) \nabla^{T} u_{k} .
$$

This is responsible for the structure of the geometric estimator (1.15). We will see below how to bound $\mathbf{A}_{k}-\mathbf{A}$ in terms of the difference of unit normals $\nu-\nu_{k}$, and thus the surface estimator (1.14). 
Lemma 2.1 (Consistency error). If $\mathbf{E}_{k} \in \mathbb{R}^{d \times d}$ is given by

$$
\mathbf{E}_{k}:=\frac{1}{q} \mathbf{T}\left(q_{k} \mathbf{D}_{k} \mathbf{D}_{k}^{T}-q \mathbf{D} \mathbf{D}^{T}\right) \mathbf{T}^{T},
$$

then for all $v, w \in H^{1}(\Omega)$,

$$
\int_{\Gamma_{k}} \nabla_{\Gamma_{k}} v \nabla_{\Gamma_{k}}^{T} w-\int_{\Gamma} \nabla_{\Gamma} v \nabla_{\Gamma}^{T} w=\int_{\Gamma} \nabla_{\Gamma} v \mathbf{E}_{k} \nabla_{\Gamma}^{T} w
$$

Proof. If $T \in \mathcal{T}_{k}\left(\Gamma_{k}\right), \hat{T} \in \mathcal{T}_{k}(\Omega)$, and $\widetilde{T} \in \mathcal{T}_{k}(\Gamma)$ are related according to (1.8), then we have with some abuse of notation $\int_{T} v w=\int_{\widehat{T}} q_{k} v w=\int_{\widetilde{T}} \frac{q_{k}}{q} v w$. Hence, in view of (2.1) and (2.5), we obtain

$$
\int_{T} \nabla_{\Gamma_{k}} v \nabla_{\Gamma_{k}}^{T} w=\int_{\widehat{T}} q_{k} \nabla_{\mathbf{x}} v \mathbf{D}_{k} \mathbf{D}_{k}^{T} \nabla_{\mathbf{x}}^{T} w=\int_{\widetilde{T}} \frac{q_{k}}{q} \nabla_{\Gamma} v\left(\mathbf{T D}_{k} \mathbf{D}_{k}^{T} \mathbf{T}^{T}\right) \nabla_{\Gamma}^{T} w .
$$

Likewise, using (2.6) and the orthogonality of $\nabla_{\Gamma} v$ and $\nu$, namely $\nabla_{\Gamma} v \mathbf{T D}=\nabla_{\Gamma} v$, we get

$$
\int_{\widetilde{T}} \nabla_{\Gamma} v \nabla_{\Gamma}^{T} w=\int_{\widetilde{T}} \nabla_{\Gamma} v \mathbf{T D D}^{T} \mathbf{T}^{T} \nabla_{\Gamma}^{T} w
$$

Subtracting these two expressions gives (2.8).

Lemma 2.2 (Basic geometric estimates). The following pointwise estimates are valid for all $k \geq 0$ :

$$
\left|q-q_{k}\right| \leq q q_{k}\left|\nu-\nu_{k}\right|, \quad\left|\frac{1}{q} \nabla z^{T} \nabla z-\frac{1}{q_{k}} \nabla z_{k}^{T} \nabla z_{k}\right| \leq 3 q q_{k}\left|\nu-\nu_{k}\right| .
$$

Proof. It follows from (1.3) that $\left|\frac{1}{q}-\frac{1}{q_{k}}\right| \leq\left|\nu-\nu_{k}\right|$, whence $\left|q-q_{k}\right| \leq q q_{k}\left|\nu-\nu_{k}\right|$. To get the last estimate, we write

$$
\begin{aligned}
\frac{\nabla z^{T} \nabla z}{q}-\frac{\nabla z_{k}{ }^{T} \nabla z_{k}}{q_{k}}= & \left(q-q_{k}\right)\left(\frac{\nabla z^{T}}{q} \frac{\nabla z}{q}\right) \\
& +q_{k}\left[\frac{\nabla z^{T}}{q}\left(\frac{\nabla z}{q}-\frac{\nabla z_{k}}{q_{k}}\right)+\left(\frac{\nabla z}{q}-\frac{\nabla z_{k}}{q_{k}}\right)^{T} \frac{\nabla z_{k}}{q_{k}}\right],
\end{aligned}
$$

and note that (1.3) implies $\left|\frac{\nabla z}{q}-\frac{\nabla z_{k}}{q_{k}}\right| \leq\left|\nu-\nu_{k}\right|$ and $\left|\frac{\nabla z}{q}\right|,\left|\frac{\nabla z_{k}}{q_{k}}\right| \leq 1$.

Lemma 2.3 (Comparing $q$ and $q_{k}$ ). If $q\left|\nu-\nu_{k}\right| \leq \frac{1}{2}$, then

$$
q_{k} \leq 2 q \leq 3 q_{k} .
$$

Proof. It suffices to write $\pm\left(q_{k}-q\right) \leq\left|q_{k}-q\right|$ and use Lemma 2.2.

The condition $\left\|q\left(\nu-\nu_{k}\right)\right\|_{L^{\infty}(\Omega)} \leq \frac{1}{2}$ entails a minimal degree of surface resolution, and is compatible with the threshold $\lambda_{k} \leq \Lambda$ leading to the contraction property of Theorem 4.1. Moreover, the equivalence $q_{k} \approx q$ is valid asymptotically with constant as close to 1 as desired.

Lemma 2.4 (Estimate of $\mathbf{E}_{k}$ ). Let $\mathbf{E}_{k}$ be defined in (2.7) and $\lambda_{k}$ in (1.14). Then, if $q\left|\nu-\nu_{k}\right| \leq \frac{1}{2}$

$$
\left\|\mathbf{E}_{k}\right\|_{L^{\infty}(T)} \leq Q\left\|\nu-\nu_{k}\right\|_{L^{\infty}(\widehat{T})}=Q \lambda_{k}(T), \quad \forall T \in \mathcal{T}_{k},
$$

with $Q:=8\|q\|_{L^{\infty}(\Omega)}^{3}$. 
Proof. In view of (2.4), we can write

$$
q_{k} \mathbf{D}_{k} \mathbf{D}_{k}^{T}-q \mathbf{D} \mathbf{D}^{T}=\left(q_{k}-q\right) \mathbf{I}-\left(\frac{1}{q_{k}} \nabla z_{k}^{T} \nabla z_{k}-\frac{1}{q} \nabla z^{T} \nabla z\right) .
$$

Since $|\mathbf{T}|=q$, the assertion follows from the definition (2.7) and Lemmas 2.2 and 2.3 ,

Remark 2.3 (Order of $\mathbf{E}_{k}$ ). The formal order of both $\mathbf{E}_{k}$ and the energy error is the same, namely the polynomial degree $n \geq 1$. If $z$ were represented via the distance function, as in 9, 10, 13, then the formal order of $\mathbf{E}_{k}$ would be $n+1$. This is due to the fact that $\mathbf{T}$ would then be a small perturbation of the orthogonal projection onto the tangent plane to $z$, whereas our $\mathbf{T}$ is just a mapping onto it. This fact is compensated by flexibility in describing the surface and is instrumental in [5].

Lemma 2.5 (Perturbation of D). The following pointwise estimate holds for all $k$

$$
\left|\mathbf{D}-\mathbf{D}_{k}\right| \leq 5 q\left|\nu-\nu_{k}\right| \text {. }
$$

Proof. Use the definition (2.2) to write

$$
\mathbf{D}-\mathbf{D}_{k}=\left(\frac{1}{q}-\frac{1}{q_{k}}\right) \frac{\nabla z^{T}}{q}(-\nabla z, 1)+\frac{1}{q_{k}}\left(\frac{\nabla z^{T}}{q}(-\nabla z, 1)-\frac{\nabla z_{k}^{T}}{q_{k}}\left(-\nabla z_{k}, 1\right)\right)
$$

and estimate each term separately using Lemma 2.2 .

Lemma 2.6 (Equivalence of norms). Let $\Gamma_{k}$ be a piecewise polynomial approximation of $\Gamma$ as described above. For all lifted functions $v: \Omega \times \mathbb{R} \rightarrow \mathbb{R}$, we have the following equivalences of local (semi)norms

$$
\|v\|_{L^{2}(\widehat{T})} \approx\|v\|_{L^{2}(T)} \approx\|v\|_{L^{2}(\widetilde{T})}, \quad\|\nabla v\|_{L^{2}(\widehat{T})} \approx\left\|\nabla_{\Gamma_{k}} v\right\|_{L^{2}(T)} \approx\left\|\nabla_{\Gamma} v\right\|_{L^{2}(\widetilde{T})},
$$

which lead to the following equivalences of the global (semi)norms

$$
\|v\|_{L^{2}(\Omega)} \approx\|v\|_{L^{2}\left(\Gamma_{k}\right)} \approx\|v\|_{L^{2}(\Gamma)}, \quad\|\nabla v\|_{L^{2}(\Omega)} \approx\left\|\nabla_{\Gamma_{k}} v\right\|_{L^{2}\left(\Gamma_{k}\right)} \approx\left\|\nabla_{\Gamma} v\right\|_{L^{2}(\Gamma)},
$$

with equivalence constants depending only on the Lipschitz constant $\|\nabla z\|_{L^{\infty}(\Omega)}$ of $z$.

Proof. Let $T \in \mathcal{T}_{k}\left(\Gamma_{k}\right), \widehat{T} \in \mathcal{T}_{k}(\Omega)$ and $\widetilde{T} \in \mathcal{T}_{k}(\Gamma)$ satisfy (1.8). The first equivalence holds trivially from the change of variables leading to

$$
\int_{\widehat{T}} q_{k}|v|^{2}=\int_{T}|v|^{2}=\int_{\widetilde{T}} \frac{q_{k}}{q}|v|^{2}
$$

and the fact that $0<c \leq \frac{q_{k}}{q} \leq q_{k} \leq C$ for constants $c, C$ depending only on $\|\nabla z\|_{L^{\infty}(\Omega)}$. To prove the second equivalence, we resort to (2.9) to write

$$
\int_{T}\left|\nabla_{\Gamma_{k}} v\right|^{2}=\int_{\widetilde{T}} \frac{q_{k}}{q} \nabla_{\Gamma} v\left(\mathbf{T D}_{k} \mathbf{D}_{k}^{T} \mathbf{T}^{T}\right) \nabla_{\Gamma}^{T} v \leq \int_{\widetilde{T}} \frac{q_{k}}{q}\left(1+5 q^{2}\left|\nu-\nu_{k}\right|\right)^{2}\left|\nabla_{\Gamma} v\right|^{2},
$$

because $\mathbf{T D}_{k}=\mathbf{T D}+\mathbf{T}\left(\mathbf{D}_{k}-\mathbf{D}\right)$ and Lemma 2.5, and analogously

$$
\int_{\widetilde{T}}\left|\nabla_{\Gamma} v\right|^{2}=\int_{T} \frac{q}{q_{k}} \nabla_{\Gamma_{k}} v\left(\mathbf{T}_{k} \mathbf{D} \mathbf{D}^{T} \mathbf{T}_{k}^{T}\right) \nabla_{\Gamma_{k}}^{T} v \leq \int_{T} \frac{q}{q_{k}}\left(1+5 q_{k}^{2}\left|\nu-\nu_{k}\right|\right)^{2}\left|\nabla_{\Gamma_{k}} v\right|^{2} .
$$

These inequalities, in conjunction with the identities (2.1) and (2.5), and the fact that $|\mathbf{D}|=1$ and $|\mathbf{T}|=q \leq 1+\|\nabla z\|_{L^{\infty}(\Omega)}$, yield the second (local) equivalence. The global equivalences are easily obtained by adding. 
We now establish inverse estimates for discrete unit normals $\nu_{k}, \nu_{k-1}$. The derivation is rather technical because discrete unit normals are not piecewise polynomials. We exploit the fact that they are written in terms of derivatives of $z_{k}, z_{k-1}$, which are piecewise polynomials and thus allow for inverse estimates. A salient feature is that we only assume Lipschitz regularity of $\Gamma$.

Lemma 2.7 (Inverse inequalities for discrete unit normals). If $k \geq 1$ and $T \in \mathcal{T}_{k}$, then

$$
\left\|D \nu_{k}\right\|_{L^{\infty}(T)} \lesssim \frac{1}{h_{T}}, \quad\left\|D\left(\nu_{k}-\nu_{k-1}\right)\right\|_{L^{\infty}(T)} \lesssim \frac{1}{h_{T}}\left\|\nu_{k}-\nu_{k-1}\right\|_{L^{\infty}(T)},
$$

where the constants involved in $\lesssim$ depend on the mesh regularity of $\mathcal{T}_{k}(\Omega)$ and $\|\nabla z\|_{L^{\infty}(\Omega)}$.

Proof. Observe first that for $x, y \in \widehat{T}$,

$$
\begin{aligned}
\mid \nu_{k}(x) & -\nu_{k}(y) \mid \\
& \leq\left|\frac{1}{q_{k}(x)}-\frac{1}{q_{k}(y)}\right|+\left|\frac{\nabla z_{k}(x)}{q_{k}(x)}-\frac{\nabla z_{k}(y)}{q(y)}\right| \\
& \leq\left|\frac{q_{k}(y)-q_{k}(x)}{q_{k}(x) q_{k}(y)}\right|+\left|\frac{q_{k}(y) \nabla z_{k}(x)-q_{k}(x) \nabla z_{k}(y)}{q_{k}(x) q_{k}(y)}\right| \\
& \leq \frac{\left|q_{k}(y)-q_{k}(x)\right|}{q_{k}(x) q_{k}(y)}+\frac{\left|\nabla z_{k}(x)-\nabla z_{k}(y)\right|}{q_{k}(x)}+\frac{\left|q_{k}(y)-q_{k}(x)\right|\left|\nabla z_{k}(y)\right|}{q_{k}(x) q_{k}(y)} .
\end{aligned}
$$

Since the mapping $v \rightarrow \sqrt{1+|v|^{2}}$ from $\mathbb{R}^{d-1} \rightarrow \mathbb{R}$ is globally Lipschitz, and $q_{k}=$ $\sqrt{1+\left|\nabla z_{k}\right|^{2}}$

$$
\begin{aligned}
\frac{\left|q_{k}(y)-q_{k}(x)\right|}{|x-y|} & \lesssim \frac{\left|\nabla z_{k}(y)-\nabla z_{k}(x)\right|}{|x-y|} \\
& \leq\left\|D^{2} z_{k}\right\|_{L^{\infty}(\widehat{T})} \lesssim \frac{\left\|\nabla z_{k}\right\|_{L^{\infty}(\widehat{T})}}{h_{T}} \lesssim \frac{1}{h_{T}}
\end{aligned}
$$

by an inverse inequality for $z_{k}$ and the fact that $\left\|\nabla z_{k}\right\|_{L^{\infty}(\widehat{T})} \lesssim\|\nabla z\|_{L^{\infty}(\Omega)} \lesssim 1$. Combining (2.15) with (2.16) and using again that $\left\|\nabla z_{k}\right\|_{L^{\infty}(\widehat{T})} \lesssim 1$ and $q_{k} \geq 1$, we conclude that

$$
\sup _{x, y \in T} \frac{\left|\nu_{k}(x)-\nu_{k}(y)\right|}{|x-y|} \lesssim \frac{1}{h_{T}}
$$

and the first assertion in (2.14) follows.

In order to prove the second assertion, we denote $\boldsymbol{\delta}[v](x)=v_{k}(x)-v_{k-1}(x)$ for a generic function $v$ such as $\nu, z, q, q^{-1}$, and observe that for $x, y \in \widehat{T}$,

$$
|\boldsymbol{\delta}[\nu](x)-\boldsymbol{\delta}[\nu](y)| \leq\left|\boldsymbol{\delta}\left[\frac{1}{q}\right](x)-\boldsymbol{\delta}\left[\frac{1}{q}\right](y)\right|+\left|\boldsymbol{\delta}\left[\frac{\nabla z}{q}\right](x)-\boldsymbol{\delta}\left[\frac{\nabla z}{q}\right](y)\right|
$$


For the first term on the right-hand side we have

$$
\begin{aligned}
\left|\boldsymbol{\delta}\left[\frac{1}{q}\right](x)-\boldsymbol{\delta}\left[\frac{1}{q}\right](y)\right| \leq & \left|\frac{\boldsymbol{\delta}[q](x) q_{k}(y) q_{k-1}(y)-\boldsymbol{\delta}[q](y) q_{k}(x) q_{k-1}(x)}{q_{k}(x) q_{k}(y) q_{k-1}(x) q_{k-1}(y)}\right| \\
\leq & \frac{1}{q_{k}(x) q_{k}(y) q_{k-1}(x) q_{k-1}(y)} \\
& \times\left\{|\boldsymbol{\delta}[q](x)|\left|q_{k}(y)-q_{k}(x)\right| q_{k-1}(y)\right. \\
& \quad+|\boldsymbol{\delta}[q](x)| q_{k}(x)\left|q_{k-1}(y)-q_{k-1}(x)\right| \\
& \left.+|\boldsymbol{\delta}[q](x)-\boldsymbol{\delta}[q](y)| q_{k}(x) q_{k-1}(x)\right\} .
\end{aligned}
$$

Now, by the first inequality of Lemma 2.2 (with $q_{k-1}$ instead of $q$ ) and (2.16),

$$
\begin{aligned}
|\boldsymbol{\delta}[q](x)|\left|q_{k}(y)-q_{k}(x)\right| & \lesssim\|\boldsymbol{\delta}[\nu]\|_{L^{\infty}(T)}\left|q_{k}(y)-q_{k}(x)\right| \\
& \lesssim\|\boldsymbol{\delta}[\nu]\|_{L^{\infty}(T)} \frac{|x-y|}{h_{T}}, \\
|\boldsymbol{\delta}[q](x)|\left|q_{k-1}(y)-q_{k-1}(x)\right| & \lesssim\|\boldsymbol{\delta}[\nu]\|_{L^{\infty}(T)}\left|q_{k-1}(y)-q_{k-1}(x)\right| \\
& \lesssim\|\boldsymbol{\delta}[\nu]\|_{L^{\infty}(T)} \frac{|x-y|}{h_{T}} .
\end{aligned}
$$

On the other hand,

$$
|\boldsymbol{\delta}[q](x)-\boldsymbol{\delta}[q](y)| \leq|y-x| \int_{0}^{1}|\nabla \boldsymbol{\delta}[q]((1-t) x+t y)| d t .
$$

Now notice that $\nabla q_{k}=\frac{1}{q_{k}} \nabla z_{k} D^{2} z_{k}$, and $\boldsymbol{\delta}\left[D^{2} z\right]=D^{2} \boldsymbol{\delta}[z], \boldsymbol{\delta}[\nabla z]=\nabla \boldsymbol{\delta}[z]$, which only involve derivatives of the discrete approximations $z_{k}$ and $z_{k-1}$, but no derivatives of $z$ which is only assumed to be $C^{1}$. Therefore, at any point in $\widehat{T}$,

$$
\begin{aligned}
& |\nabla \boldsymbol{\delta}[q]|=|\boldsymbol{\delta}[\nabla q]|=\left|\boldsymbol{\delta}\left[\frac{1}{q} \nabla z D^{2} z\right]\right| \\
& \quad \leq\left|\boldsymbol{\delta}\left[\frac{1}{q}\right]\right|\left|\nabla z_{k-1}\right|\left|D^{2} z_{k-1}\right|+\left|\frac{1}{q_{k}}\right||\boldsymbol{\delta}[\nabla z]|\left|D^{2} z_{k-1}\right|+\left|\frac{1}{q_{k}}\right|\left|\nabla z_{k}\right|\left|\boldsymbol{\delta}\left[D^{2} z\right]\right| \\
& \quad \lesssim\|\boldsymbol{\delta}[\nu]\|_{L^{\infty}(T)} \frac{\left\|\nabla z_{k-1}\right\|_{L^{\infty}(T)}^{2}}{h_{T}}+\frac{\left\|\nabla z_{k-1}\right\|_{L^{\infty}(T)}+\left\|\nabla z_{k}\right\|_{L^{\infty}(T)}}{h_{T}}\|\boldsymbol{\delta}[\nabla z]\|_{L^{\infty}(T)},
\end{aligned}
$$

where in the last inequality we have used inverse inequalities for $z_{k}, z_{k-1}, \boldsymbol{\delta}[z]$, and that $q_{k} \geq 1$. Using again the first inequality of Lemma 2.2, and (2.16), gives

$$
\begin{aligned}
\|\boldsymbol{\delta}[\nabla z]\|_{L^{\infty}(T)}=\|\boldsymbol{\delta}[q \nu]\|_{L^{\infty}(T)} \leq\left\|\boldsymbol{\delta}[q] \nu_{k}\right\|_{L^{\infty}(T)} \\
+\left\|q_{k-1} \boldsymbol{\delta}[\nu]\right\|_{L^{\infty}(T)} \lesssim\|\boldsymbol{\delta}[\nu]\|_{L^{\infty}(T)},
\end{aligned}
$$

whence the three previous estimates yield

$$
|\boldsymbol{\delta}[q](x)-\boldsymbol{\delta}[q](y)| \lesssim \frac{\|\boldsymbol{\delta}[\nu]\|_{L^{\infty}(T)}}{h_{T}}|x-y| .
$$

Inserting (2.20), (2.21) and (2.23) into (2.19) we obtain

$$
\frac{\left|\boldsymbol{\delta}\left[\frac{1}{q}\right](x)-\boldsymbol{\delta}\left[\frac{1}{q}\right](y)\right|}{|x-y|} \lesssim \frac{\|\boldsymbol{\delta}[\nu]\|_{L^{\infty}(T)}}{h_{T}} .
$$


We bound the second term in the right-hand side of (2.18) as follows

$$
\begin{aligned}
\left|\boldsymbol{\delta}\left[\frac{\nabla z}{q}\right](x)-\boldsymbol{\delta}\left[\frac{\nabla z}{q}\right](y)\right| \\
\leq\left|\frac{\boldsymbol{\delta}[\nabla z](x)}{q_{k-1}(x)}-\frac{\boldsymbol{\delta}[\nabla z](y)}{q_{k-1}(y)}\right|+\left|\nabla z_{k}(x) \boldsymbol{\delta}\left[\frac{1}{q}\right](x)-\nabla z_{k}(y) \boldsymbol{\delta}\left[\frac{1}{q}\right](y)\right| \\
\leq\left|\frac{\boldsymbol{\delta}[\nabla z](x)-\boldsymbol{\delta}[\nabla z](y)}{q_{k-1}(x)}\right|+|\boldsymbol{\delta}[\nabla z](y)|\left|\frac{1}{q_{k-1}(x)}-\frac{1}{q_{k-1}(y)}\right| \\
\quad+\left|\nabla z_{k}(x)\right|\left|\boldsymbol{\delta}\left[\frac{1}{q}\right](x)-\boldsymbol{\delta}\left[\frac{1}{q}\right](y)\right|+\left|\nabla z_{k}(x)-\nabla z_{k}(y)\right|\left|\boldsymbol{\delta}\left[\frac{1}{q}\right](y)\right| .
\end{aligned}
$$

We now bound all the terms on the right-hand side of (2.25). An inverse inequality for $\boldsymbol{\delta}[z]$ and (2.22) lead to the following bound for the first term:

$$
\begin{aligned}
\left|\frac{\boldsymbol{\delta}[\nabla z](x)-\boldsymbol{\delta}[\nabla z](y)}{q_{k-1}(x)}\right| & \lesssim\left\|\boldsymbol{\delta}\left[D^{2} z\right]\right\|_{L^{\infty}(T)}|x-y| \\
& \lesssim \frac{\|\boldsymbol{\delta}[\nabla z]\|_{L^{\infty}(T)}}{h_{T}}|x-y| \lesssim \frac{\|\boldsymbol{\delta}[\nu]\|_{L^{\infty}(T)}}{h_{T}}|x-y|,
\end{aligned}
$$

where we have used again that $\boldsymbol{\delta}\left[D^{2} z\right]=D^{2} \boldsymbol{\delta}[z]$ and $\boldsymbol{\delta}[\nabla z]=\nabla \boldsymbol{\delta}[z]$. In view of (2.22) and (2.17), the second term on the right-hand side of (2.25) is bounded as follows:

$$
\begin{aligned}
|\boldsymbol{\delta}[\nabla z](y)|\left|\frac{1}{q_{k-1}(x)}-\frac{1}{q_{k-1}(y)}\right| & \lesssim\|\boldsymbol{\delta}[\nu]\|_{L^{\infty}(T)}\left|\nu_{k-1}(x)-\nu_{k-1}(y)\right| \\
& \lesssim \frac{\|\boldsymbol{\delta}[\nu]\|_{L^{\infty}(T)}}{h_{T}}|x-y| .
\end{aligned}
$$

By (2.24), the third term satisfies

$$
\left|\nabla z_{k}(x)\right|\left|\boldsymbol{\delta}\left[\frac{1}{q}\right](x)-\boldsymbol{\delta}\left[\frac{1}{q}\right](y)\right| \lesssim \frac{\|\boldsymbol{\delta}[\nu]\|_{L^{\infty}(T)}}{h_{T}}|x-y| .
$$

Finally, the fourth term satisfies

$$
\begin{aligned}
\left|\nabla z_{k}(x)-\nabla z_{k}(y)\right|\left|\boldsymbol{\delta}\left[\frac{1}{q}\right](y)\right| & \lesssim\left\|D^{2} z_{k}\right\|_{L^{\infty}(\widehat{T})}|x-y|\|\boldsymbol{\delta}[\nu]\|_{L^{\infty}(T)} \\
& \lesssim \frac{\|\boldsymbol{\delta}[\nu]\|_{L^{\infty}(T)}}{h_{T}}|x-y|,
\end{aligned}
$$

by an inverse inequality for $z_{k}$. Inserting the above estimates into (2.25) we obtain

$$
\frac{\left|\boldsymbol{\delta}\left[\frac{\nabla z}{q}\right](x)-\boldsymbol{\delta}\left[\frac{\nabla z}{q}\right](y)\right|}{|x-y|} \lesssim \frac{\|\boldsymbol{\delta}[\nu]\|_{L^{\infty}(T)}}{h_{T}} .
$$

Combining (2.18) with (2.24) and (2.26), and recalling the definition of $\delta[\cdot]$ we obtain

$$
\sup _{x, y \in T} \frac{\left|\left[\nu_{k}(x)-\nu_{k-1}(x)\right]-\left[\nu_{k}(y)-\nu_{k-1}(y)\right]\right|}{|x-y|} \lesssim \frac{\left\|\nu_{k}-\nu_{k-1}\right\|_{L^{\infty}(T)}}{h_{T}},
$$

which readily implies the second assertion in (2.14). 


\section{AFEM: Main MODUles AND ALGORIThM}

In this section we discuss the main modules in the adaptive loop (1.10) along with AFEM.

3.1. Procedure SOLVE. This procedure solves the SPD linear system resulting from (1.9). We employ either a direct solver or any standard iterative solver, such as conjugate gradient (CG) with hierarchical basis or BPX preconditioning. Given an approximate surface-mesh pair $\left(\Gamma_{k}, \mathcal{T}_{k}\right)$, a forcing function $F_{k}$, and an initial guess for the solution $U_{k-1}$, SOLVE computes the discrete solution

$$
U_{k}:=\operatorname{SOLVE}\left(\Gamma_{k}, \mathcal{T}_{k}, F_{k}, U_{k-1}\right) \text {. }
$$

We assume, for simplicity, exact linear algebra and quadrature in computing $U_{k}$.

3.2. Procedure ESTIMATE. This procedure computes a posteriori error estimators that account for both PDE and geometry. We first need to find an error representation formula. Subtracting the weak formulations (1.7) and (1.9), and integrating by parts elementwise, we obtain for all $v \in H_{0}^{1}(\Gamma)$ and $V \in \stackrel{\circ}{\mathbb{V}}_{k}$,

$$
\int_{\Gamma} \nabla_{\Gamma}\left(u-U_{k}\right) \cdot \nabla_{\Gamma} v=I_{1}+I_{2}+I_{3}
$$

with

$$
\begin{aligned}
I_{1} & :=\sum_{T \in \mathcal{T}_{k}} \int_{T} \mathcal{R}\left(U_{k}\right)(v-V)-\sum_{S \in \mathcal{S}_{k}^{o}} \int_{S} \mathcal{J}\left(U_{k}\right)(v-V), \\
I_{2} & :=\int_{\Gamma_{k}} \nabla_{\Gamma_{k}} U_{k} \cdot \nabla_{\Gamma_{k}} v-\int_{\Gamma} \nabla_{\Gamma} U_{k} \cdot \nabla_{\Gamma} v=\int_{\Gamma} \nabla_{\Gamma} U_{k} \mathbf{E}_{k} \nabla_{\Gamma}^{T} v, \\
I_{3} & :=\int_{\Gamma} f v-\int_{\Gamma_{k}} F_{k} v .
\end{aligned}
$$

To prove an upper bound of the error in terms of the a posteriori error indicators, we need an $H_{0}^{1}(\Gamma)$-stable quasi-interpolation operator $\mathcal{I}_{k}: H_{0}^{1}(\Gamma) \rightarrow \mathbb{V}_{k}$, such as those of Clemént or Scott-Zhang [3, 7. We proceed as in 8 8 to construct $\mathcal{I}_{k}$ on $\mathcal{T}_{k}(\Omega)$, which is shape-regular, and lift it on $\Gamma$ and $\Gamma_{k}$ via (1.2). Lemma 2.6 implies for all $v \in H_{0}^{1}(\Gamma)$, and all $T \in \mathcal{T}_{k}\left(\Gamma_{k}\right)$

$$
h_{T}^{-1}\left\|v-\mathcal{I}_{k} v\right\|_{L^{2}(T)}+h_{T}^{-1 / 2}\left\|v-\mathcal{I}_{k} v\right\|_{L^{2}(\partial T)} \lesssim\left\|\nabla_{\Gamma} v\right\|_{L^{2}\left(N_{k}(T)\right)},
$$

where $h_{T}$ denotes the diameter of $T$ and $N_{k}(T):=\bigcup\left\{T^{\prime} \in \mathcal{T}_{k}\left(\Gamma_{k}\right) \mid T^{\prime} \cap T \neq \emptyset\right\}$. The constant behind $\lesssim$ depends on the shape regularity of $\mathcal{T}_{k}(\Omega)$ and the Lipschitz constant $\|\nabla z\|_{L^{\infty}(\Omega)}$ of $z$.

Lemma 3.1 (Upper bound). Let the estimators $\eta_{k}, \zeta_{k}$ be given by (1.13) and (1.15), and let $F_{k}$ be

$$
F_{k}\left(\mathbf{x}, z_{k}(\mathbf{x})\right):=\frac{q(\mathbf{x})}{q_{k}(\mathbf{x})} f(\mathbf{x}, z(\mathbf{x})) .
$$

Then there exists a constant $C_{1}$, depending only on the shape regularity of $\mathcal{T}_{k}(\Omega)$ and $\|\nabla z\|_{L^{\infty}(\Omega)}$, such that

$$
e_{k}^{2} \leq C_{1}\left(\eta_{k}^{2}+\zeta_{k}^{2}\right)
$$


Proof. Since (3.3) implies $I_{3}=0$ in (3.1), it remains to deal with $I_{1}$ and $I_{2}$. Taking $v=u-U_{k} \in H_{0}^{1}(\Gamma), V=\mathcal{I}_{k} v \in \stackrel{\mathbb{V}}{k}_{k}$ into (3.1), and using (3.2) and (1.13), we arrive at

$$
e_{k}^{2} \lesssim \sum_{T \in \mathcal{T}_{k}} \eta_{k}^{2}(T)+\sum_{T \in \mathcal{T}_{k}}\left\|\nabla_{\Gamma} U_{k} \mathbf{E}_{k}\right\|_{L^{2}(\widetilde{T})}^{2} .
$$

Finally, recalling (1.14) and (1.15), along with (2.12), we end up with (3.4).

We point out that a choice similar to (3.3) is made in [10; its main purpose is to yield $I_{3}=0$ and thereby simplify the analysis. Other choices of $F_{k}$, such as a lifting of $f$, would introduce additional oscillation terms that could be handled accordingly.

In contrast to 11, 15, 16, 17, 18, our subsequent convergence analysis does not need a lower bound, and we thus omit it. However, such a bound may be crucial for optimality [4.

Given an approximate surface-mesh pair $\left(\Gamma_{k}, \mathcal{T}_{k}\right)$, a forcing function $F_{k}$, and a discrete solution $U_{k}$, (3.4) reveals that the behavior of the energy error $e_{k}$ is governed directly by the two local error indicators $\eta_{k}(T), \zeta_{k}(T)$, and indirectly by $\lambda_{k}(T)$ due to definition (1.15). The procedure ESTIMATE computes these quantities, along with $\rho_{k}(T)$ given by (1.17):

$$
\left\{\eta_{k}(T), \zeta_{k}(T), \lambda_{k}(T), \rho_{k}(T)\right\}_{T \in \mathcal{T}_{k}}:=\operatorname{ESTIMATE}\left(\Gamma, \Gamma_{k}, \mathcal{T}_{k}, F_{k}, U_{k}\right)
$$

3.3. Procedure MARK. Let $\beta_{1}, \beta_{2}$ be two constants to be determined later, let $\mathcal{E}_{k}(T)$ be the total error indicator given by

$$
\mathcal{E}_{k}^{2}(T):=\eta_{k}^{2}(T)+\beta_{1} \zeta_{k}^{2}(T)+\beta_{2} \rho_{k}^{2}(T),
$$

and let $\mathcal{E}_{k}:=\left(\sum_{T \in \mathcal{T}_{k}} \mathcal{E}_{k}^{2}(T)\right)^{1 / 2}$. Given a surface-mesh pair $\left(\Gamma_{k}, \mathcal{T}_{k}\right)$, MARK enforces the following marking of $\mathcal{T}_{k}$ : Given the parameter $0<\theta<1$, the set of marked elements $\mathcal{M}_{k}$ must satisfy

$$
\mathcal{E}_{k}\left(\mathcal{M}_{k}\right) \geq \theta \mathcal{E}_{k}\left(\mathcal{T}_{k}\right)
$$

Marking (3.6) uses Dorfler's strategy [11, 15, 16, 17, 18] and forces reduction of $\mathcal{E}_{k}$ as shown in Lemmas 4.2 and 4.1. We refer to this procedure as

$$
\mathcal{M}_{k}:=\operatorname{MARK}\left(\mathcal{T}_{k},\left\{\mathcal{E}_{k}(T)\right\}_{T \in \mathcal{T}_{k}}\right) \text {. }
$$

In spite of dealing with scaling constants $\beta_{1}, \beta_{2}$, we avoid separate Dörfler marking by each estimator $\eta_{k}, \zeta_{k}, \rho_{k}$ [15, 16, 17, 18. In fact, this may lead to suboptimal meshes according to [4].

3.4. Procedure REFINE. We start this procedure with an approximate surfacemesh pair $\left(\Gamma_{0}, \mathcal{T}_{0}\right)$ that is suitably labeled, and use bisection with precise rules, such as newest vertex bisection for $d=3$, to preserve mesh regularity. We thus bisect an element of the approximate surface $\Gamma_{k}$ and then lift the new node from $\Gamma_{k}$ to $\Gamma$ along the (vertical) $x_{d}$-axis. If $\left(\mathbf{x}, z_{k}(\mathbf{x})\right) \in \Gamma_{k}$ is a new node obtained by refining $T \in \mathcal{T}_{k}$, then it is lifted to $(\mathbf{x}, z(\mathbf{x})) \in \Gamma$ to become a new node of $\Gamma_{k+1}$; see Figures 1 and 2. The new discrete surface $\Gamma_{k+1}$ is formed by interpolating new nodes and old nodes via $z_{k+1}=\mathcal{I}_{k+1} z \in \mathbb{V}_{k+1}$. Since $\Gamma$ is $C^{1}$, the new surface $\Gamma_{k+1}$ is a better piecewise polynomial approximation of $\Gamma$ than $\Gamma_{k}$ asymptotically: if $\mathcal{T}_{k+1}(T)$ denotes the set of elements $T^{\prime} \in \mathcal{T}_{k+1}$ such that $\widehat{T}^{\prime} \subset \widehat{T}$, then $\lambda_{k+1}\left(T^{\prime}\right) \leq \lambda_{k}(T)$ 
for all $T^{\prime} \in \mathcal{T}_{k+1}(T)$, whence $\lambda_{k}(T)$ is monotone. However, in the pre-asymptotic regime it may happen that monotonicity fails.

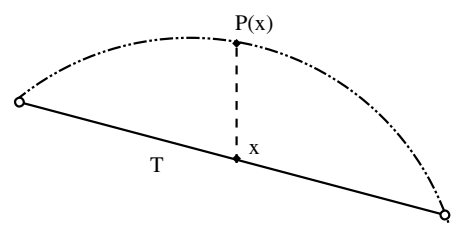

FIGURE 1. The element $T \in \mathcal{T}_{k}$ is bisected thereby giving rise to the new node $x$. This node is lifted (projected) to $P(x) \in \Gamma$ along the vertical axis.

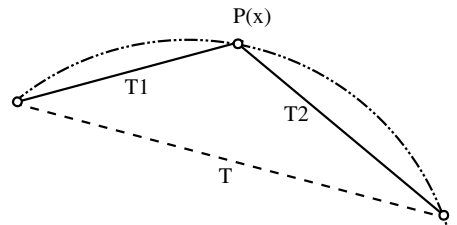

Figure 2. Two new elements $T 1$ and $T 2$ in $\mathcal{T}_{k+1}$ are formed joining the new node $P(x) \in \Gamma$ with the old nodes in $\Gamma_{k}$.

The design of REFINE takes this observation into account. REFINE bisects each marked element in $\mathcal{M}_{k}$ at least $b \geq 1$ times and keeps the mesh conforming. This may lead to additional refinement of surrounding elements. Moreover, REFINE enforces an increasingly better surface approximation according to the following two criteria: given a parameter $\xi<1$, REFINE guarantees that

$$
\begin{gathered}
\lambda_{k+1}\left(T^{\prime}\right) \leq \xi \lambda_{k}(T) \quad \forall T \in \mathcal{M}_{k}, \quad \forall T^{\prime} \in \mathcal{T}_{k+1}(T), \\
\lambda_{k+1}\left(T^{\prime}\right) \leq \lambda_{k}(T) \quad \forall T \in \mathcal{T}_{k} \backslash \mathcal{M}_{k}, \quad \forall T^{\prime} \in \mathcal{T}_{k+1}(T),
\end{gathered}
$$

to obtain a new (finer) approximate surface-mesh pair $\left(\Gamma_{k+1}, \mathcal{T}_{k+1}\right)$. We refer to this procedure as

$$
\left(\mathcal{T}_{k+1}, \Gamma_{k+1}\right):=\operatorname{REFINE}\left(\mathcal{T}_{k}, \mathcal{M}_{k},\left\{\lambda_{k}(T)\right\}_{T \in \mathcal{T}_{k}}\right) .
$$

As a consequence of (3.7) and (3.8), the surface estimator $\lambda_{k}$ satisfies the monotonicity property

$$
\lambda_{k+1} \leq \lambda_{k}, \quad \forall k \geq 0 .
$$

Remark 3.1 (Regularity of $\Gamma$ ). Since $\Gamma$ is $C^{1}$, both (3.7) and (3.8) can be achieved in finite steps. Moreover, if $\Gamma$ were $C^{m+1}$ with $1 \leq m \leq n$ (the polynomial degree), then $\lambda_{k}(T)$ would reduce proportionally to $h_{T}^{m}$, with $h_{T}$ being the diameter of $T \in \mathcal{T}_{k}$.

Remark 3.2 (Choice of $\xi$ ). If $\Gamma$ is $C^{m+1}$ with $1 \leq m \leq n$, then asymptotically we have $\lambda_{k+1}\left(T^{\prime}\right) / \lambda_{k}(T) \approx h_{T^{\prime}}^{m} / h_{T}^{m} \leq 2^{-b m /(d-1)}$, whence $\xi \geq 2^{-b m /(d-1)}$ gives a natural lower bound for $\xi$. With this choice, bisection alone guarantees the validity of (3.7) asymptotically.

Remark 3.3 (Interior node property). This refinement strategy avoids the interior node property of Morin et al. [16, 17, 18, 15]. This is quite convenient for implementation, especially for $d=4$ because 6 bisections would be needed otherwise. This substantial improvement was first proposed in 4 for flat domains.

3.5. Adaptive algorithm: AFEM. We choose parameters $0<\theta, \xi<1$, and let $U_{-1}=0$. The adaptive algorithm consists of looping through the procedures SOLVE, ESTIMATE, MARK, and REFINE as follows: 


\section{AFEM}

(1) Pick an initial approximate surface-mesh pair $\left(\Gamma_{0}, \mathcal{T}_{0}\right)$ and set $k=0$;

(2) $U_{k}=\operatorname{SOLVE}\left(\Gamma_{k}, \mathcal{T}_{k}, F_{k}, U_{k-1}\right)$;

(3) $\left\{\eta_{k}(T), \zeta_{k}(T), \lambda_{k}(T), \rho_{k}(T)\right\}_{T \in \mathcal{T}_{k}}=\operatorname{ESTIMATE}\left(\Gamma, \Gamma_{k}, \mathcal{T}_{k}, F_{k}, U_{k}\right)$;

(4) $\mathcal{M}_{k}=\operatorname{MARK}\left(\left\{\eta_{k}(T), \zeta_{k}(T), \lambda_{k}(T), \rho_{k}(T)\right\}_{T \in \mathcal{T}_{k}}\right)$;

(5) $\left(\mathcal{T}_{k+1}, \Gamma_{k+1}\right)=\operatorname{REFINE}\left(\mathcal{T}_{k}, \mathcal{M}_{k},\left\{\lambda_{k}(T)\right\}_{T \in \mathcal{T}_{k}}\right)$;

(6) Set $k=k+1$ and go to Step 2.

\section{Convergence of AFEM}

In this section we derive several crucial properties of AFEM and use them to prove convergence. Throughout this section we will use the notation

$$
e_{k}(\omega):=\left\|\nabla_{\Gamma}\left(u-U_{k}\right)\right\|_{L^{2}(\omega)}, \quad E_{k}(\omega):=\left\|\nabla_{\Gamma}\left(U_{k+1}-U_{k}\right)\right\|_{L^{2}(\omega)},
$$

for any subdomain $\omega$ of $\Gamma$ made of elements in $\mathcal{T}_{k}(\Gamma)$, and write $e_{k}, E_{k}$ whenever $\omega=\Gamma$.

4.1. Basic properties of AFEM. Recall first that $0<\theta, \xi<1$ are the user's parameters of AFEM. We start with a perturbation result for $\zeta_{k}$, which reveals a strict reduction between consecutive steps provided $E_{k}$ vanishes.

Lemma 4.1 (Reduction of geometric estimator). If $\mu_{1}:=1-\xi^{2}$ and $\delta>0$ is arbitrary, then

$$
\zeta_{k+1}^{2} \leq(1+\delta)\left(\zeta_{k}^{2}-\mu_{1} \zeta_{k}^{2}\left(\mathcal{M}_{k}\right)\right)+\left(1+\delta^{-1}\right) \lambda_{k}^{2} E_{k}^{2}
$$

Proof. Invoking definition (1.15) and Young's inequality with parameter $\delta>0$, we have

$$
\begin{aligned}
\zeta_{k+1}^{2}(T) & :=\sum_{T^{\prime} \in \mathcal{T}_{k+1}(T)} \zeta_{k+1}^{2}\left(T^{\prime}\right) \\
& \leq \lambda_{k+1}^{2}(T)\left((1+\delta)\left\|\nabla_{\Gamma} U_{k}\right\|_{L^{2}(\widetilde{T})}^{2}+\left(1+\delta^{-1}\right) E_{k}^{2}(\widetilde{T})\right), \quad \forall T \in \mathcal{T}_{k} ;
\end{aligned}
$$

recall that $\mathcal{T}_{k+1}(T)$ stands for the set of elements $T^{\prime} \in \mathcal{T}_{k+1}$ such that $\widehat{T}^{\prime} \subset \widehat{T}$. We now fix $T \in \mathcal{T}_{k}$ and distinguish two cases. Suppose first that $T \in \mathcal{M}_{k}$ and use (3.7) to deduce

$$
\zeta_{k+1}^{2}(T) \leq(1+\delta) \xi^{2} \zeta_{k}^{2}(T)+\left(1+\delta^{-1}\right) \lambda_{k+1}^{2} E_{k}^{2}(\widetilde{T}) .
$$

Suppose next that $T \notin \mathcal{M}_{k}$ and use (3.8) to infer that

$$
\zeta_{k+1}^{2}(T) \leq(1+\delta) \zeta_{k}^{2}(T)+\left(1+\delta^{-1}\right) \lambda_{k+1}^{2} E_{k}^{2}(\widetilde{T})
$$

To obtain (4.1) we simply add over $T \in \mathcal{T}_{k}$ and observe that the first terms on the right-hand sides of the two previous expressions add up to

$$
\xi^{2} \zeta_{k}^{2}\left(\mathcal{M}_{k}\right)+\zeta_{k}^{2}\left(\mathcal{T}_{k} \backslash \mathcal{M}_{k}\right)=\zeta_{k}^{2}\left(\mathcal{T}_{k}\right)-\left(1-\xi^{2}\right) \zeta_{k}^{2}\left(\mathcal{M}_{k}\right)
$$

The proof is thus complete upon setting $\mu_{1}=1-\xi^{2}$.

Lemma 4.2 (Reduction of energy error estimator). If $\mu_{2}=1-2^{\frac{b}{d-1}}$ and $\delta>0$ is arbitrary, then there exists a positive constant $C_{2}$ so that

$$
\eta_{k+1}^{2} \leq(1+\delta)\left(\eta_{k}^{2}-\mu_{2} \eta_{k}^{2}\left(\mathcal{M}_{k}\right)\right)+\left(1+\delta^{-1}\right) C_{2}\left(E_{k}^{2}+\zeta_{k}^{2}+\rho_{k}^{2}\right),
$$


where $C_{2}$ depends on the mesh regularity of $\mathcal{T}_{k}(\Omega),\|\nabla z\|_{L^{\infty}(\Omega)}$ and the polynomial degree $n$.

Proof. This proof is similar to that of Lemma 4.1 with $\lambda_{k}(T)$ replaced by $h_{T}=$ $|\widehat{T}|^{\frac{1}{d-1}}$. There are, however, some tricky details due to the fact that the operator changes with refinement. Observe that if $T^{\prime} \in \mathcal{T}_{k+1}(T)$, then

$$
\begin{aligned}
\left\|\Delta_{k+1} U_{k+1}+F_{k+1}\right\|_{L^{2}\left(\widehat{T}^{\prime}\right)} \leq & \left\|\Delta_{k} U_{k}+F_{k}\right\|_{L^{2}\left(\widehat{T}^{\prime}\right)}+\left\|\Delta_{k+1}\left(U_{k+1}-U_{k}\right)\right\|_{L^{2}\left(\widehat{T}^{\prime}\right)} \\
& +\left\|\Delta_{k+1} U_{k}-\Delta_{k} U_{k}\right\|_{L^{2}\left(\widehat{T}^{\prime}\right)}+\left\|F_{k}-F_{k+1}\right\|_{L^{2}\left(\widehat{T}^{\prime}\right)} .
\end{aligned}
$$

Now, recall the expression (1.6) for the Laplace-Beltrami operator, namely

$$
\Delta_{k+1} V=\Delta \widetilde{V}-\left(\nabla \widetilde{V} \cdot \nu_{k+1}\right)\left(\operatorname{div} \nu_{k+1}\right)-\nu_{k+1} D^{2} \widetilde{V} \nu_{k+1}^{T},
$$

along with the fact that derivatives of $\widetilde{V}$ are either derivatives of $\widehat{V}$ in $\Omega$ or zero. Applying this with $V=U_{k+1}-U_{k}$, and using an inverse inequality, together with (2.5) and (2.11) we get

$$
\left\|\Delta_{k+1}\left(U_{k+1}-U_{k}\right)\right\|_{L^{2}\left(\widehat{T}^{\prime}\right)} \lesssim \frac{1}{h_{T^{\prime}}} E_{k}(T) ;
$$

here we have also used the estimate $\left\|\operatorname{div} \nu_{k+1}\right\|_{L^{\infty}\left(T^{\prime}\right)} \lesssim \frac{1}{h_{T^{\prime}}}$ from Lemma 2.7 Again by (1.6),

$$
\begin{aligned}
\left\|\Delta_{k+1} U_{k}-\Delta_{k} U_{k}\right\|_{L^{2}\left(\widehat{T}^{\prime}\right)} & \\
= & \|\left(\Delta U_{k}-\left(\nabla U_{k} \cdot \nu_{k}\right)\left(\operatorname{div} \nu_{k}\right)-\nu_{k} D^{2} U_{k} \nu_{k}^{T}\right) \\
& \quad-\left(\Delta U_{k}-\left(\nabla U_{k} \cdot \nu_{k+1}\right)\left(\operatorname{div} \nu_{k+1}\right)-\nu_{k+1} D^{2} U_{k} \nu_{k+1}^{T}\right) \|_{L^{2}\left(\widehat{T}^{\prime}\right)} \\
\lesssim & \frac{1}{h_{T^{\prime}}}\left\|\nu_{k}-\nu_{k+1}\right\|_{L^{\infty}\left(T^{\prime}\right)}\left\|\nabla_{\Gamma} U_{k}\right\|_{L^{2}(\widetilde{T})} \leq \frac{1}{h_{T^{\prime}}} \zeta_{k}\left(T^{\prime}\right),
\end{aligned}
$$

due to an inverse inequality for $D^{2} U_{k}$, (3.7) Finally,

$$
\begin{aligned}
\left\|F_{k}-F_{k+1}\right\|_{L^{2}\left(\widehat{T}^{\prime}\right)} & =\left\|\left(\frac{q}{q_{k}}-\frac{q}{q_{k+1}}\right) f\right\|_{L^{2}\left(\widehat{T}^{\prime}\right)} \\
& \lesssim\left\|\nu_{k}-\nu_{k+1}\right\|_{L^{\infty}\left(T^{\prime}\right)}\|f\|_{L^{2}\left(\widehat{T}^{\prime}\right)} \lesssim \lambda_{k}\left(T^{\prime}\right)\|f\|_{L^{2}\left(\widehat{T}^{\prime}\right)},
\end{aligned}
$$

again by (3.7)-(3.8). We thus obtain the following estimate to be used in conjunction with (1.13)

$$
\begin{aligned}
h_{T^{\prime}}^{2} \| \Delta_{k+1} U_{k+1}+ & F_{k+1}\left\|_{L^{2}\left(\widehat{T}^{\prime}\right)}^{2} \leq(1+\delta) h_{T^{\prime}}^{2}\right\| \Delta_{k} U_{k}+F_{k} \|_{L^{2}\left(\widehat{T}^{\prime}\right)}^{2} \\
& +C\left(1+\delta^{-1}\right)\left(E_{k}^{2}\left(\widetilde{T}^{\prime}\right)+\zeta_{k}^{2}\left(T^{\prime}\right)+h_{T^{\prime}}^{2} \lambda_{k}^{2}\left(T^{\prime}\right)\|f\|_{L^{2}\left(\widehat{T}^{\prime}\right)}^{2}\right) .
\end{aligned}
$$

Now, for $S^{\prime} \in \mathcal{S}_{k+1}^{o}$, we examine the jump term $\left.\mathcal{J}_{k+1}\left(U_{k+1}\right)\right|_{S^{\prime}}$. If $T^{++}, T^{\prime-}, \mathbf{n}_{k+1}^{+}$, $\mathbf{n}_{k+1}^{-}$denote the two elements of $\mathcal{T}_{k+1}$ sharing $S^{\prime}$ and their side normals tangent to $\Gamma_{k+1}$, respectively, then the expression (1.12) for $\left.\mathcal{J}_{k+1}\left(U_{k+1}\right)\right|_{S^{\prime}}$ can be rewritten as follows for each $x \in S^{\prime}$ :

$$
\left.\left|\mathcal{J}_{k+1}\left(U_{k+1}\right)\right|_{S^{\prime}}|=| \nabla_{k+1} U_{k+1}\right|_{T^{\prime+}}-\left.\nabla_{k+1} U_{k+1}\right|_{T^{\prime-}} \mid .
$$


This is due to the fact that the component of $\nabla_{k+1} U_{k+1}$ tangential to $S^{\prime}$ does not jump because $U_{k+1}$ is globally continuous. Thus

$$
\begin{aligned}
\left|\mathcal{J}_{k+1}\left(U_{k+1}\right)\right|_{S^{\prime}} \mid \leq & \left.\left|\nabla_{k+1}\left(U_{k+1}-U_{k}\right)\right|_{T^{\prime+}}|+| \nabla_{k+1}\left(U_{k+1}-U_{k}\right)\right|_{T^{\prime-}} \mid \\
& +\left|\nabla_{k+1} U_{k}\right|_{T^{\prime}}-\left.\left.\nabla_{k} U_{k}\right|_{T^{\prime}}|+| \nabla_{k+1} U_{k}\right|_{T^{\prime}}-\left.\nabla_{k} U_{k}\right|_{T^{\prime}} \mid \\
& +\left|\nabla_{k} U_{k}\right|_{T^{\prime+}}-\left.\nabla_{k} U_{k}\right|_{T^{\prime}} \mid .
\end{aligned}
$$

Therefore, using a scaled trace theorem and inverse inequalities,

$$
\begin{aligned}
\left\|\mathcal{J}_{k+1}\left(U_{k+1}\right)\right\|_{L^{2}\left(\partial \widehat{T}^{\prime}\right)} \leq & \frac{C}{h_{T^{\prime}}^{1 / 2}}\left\|\nabla_{k+1}\left(U_{k+1}-U_{k}\right)\right\|_{L^{2}\left(\omega_{k+1}\left(\widehat{T}^{\prime}\right)\right)} \\
& +\frac{C}{h_{T^{\prime}}^{1 / 2}}\left\|\nabla_{k+1} U_{k}-\nabla_{k} U_{k}\right\|_{L^{2}\left(\omega_{k+1}\left(\widehat{T}^{\prime}\right)\right)} \\
& +\left\|\mathcal{J}_{k}\left(U_{k}\right)\right\|_{L^{2}\left(\partial \widehat{T}^{\prime}\right)},
\end{aligned}
$$

with a constant $C$ depending only on mesh regularity of $\mathcal{T}_{k}(\Omega),\|\nabla z\|_{L^{\infty}(\Omega)}$, and the polynomial degree $n$. Using Young's inequality with parameter $\delta>0$ we have that

$$
\begin{aligned}
h_{T^{\prime}}\left\|\mathcal{J}_{k+1}\left(U_{k+1}\right)\right\|_{L^{2}\left(\partial \widehat{T}^{\prime}\right)}^{2} \leq & (1+\delta) h_{T^{\prime}}\left\|\mathcal{J}_{k}\left(U_{k}\right)\right\|_{L^{2}\left(\partial \widehat{T}^{\prime}\right)}^{2} \\
& +C\left(1+\delta^{-1}\right)\left(E_{k}^{2}(T)+\zeta_{k}^{2}(T)\right) .
\end{aligned}
$$

Adding (4.4) and (4.5) over $T^{\prime} \in \mathcal{T}_{k+1}(T)$ we obtain

$$
\begin{aligned}
\eta_{k+1}^{2}(T) & :=\sum_{T^{\prime} \in \mathcal{T}_{k+1}(T)} \eta_{k+1}^{2}\left(T^{\prime}\right) \\
& \leq \max _{T^{\prime} \in \mathcal{T}_{k+1}(T)} \frac{h_{T^{\prime}}}{h_{T}}(1+\delta) \eta_{k}^{2}\left(U_{k}, T\right)+C\left(1+\delta^{-1}\right)\left[E_{k}^{2}(T)+\zeta_{k}^{2}(T)+\rho_{k}^{2}\right],
\end{aligned}
$$

with $\rho_{k}^{2}=\sum_{T \in \mathcal{T}_{k}} \lambda_{k}^{2}(T) h_{k+1}(T)^{2}\|f\|_{L^{2}(T)}^{2}$. Since the mesh-size obeys MARK and REFINE we see that for all $T^{\prime} \in \mathcal{T}_{k+1}(T)$,

$$
h_{T^{\prime}} \leq \begin{cases}2^{-\frac{b}{d-1}} h_{T} & \text { if } T \in \mathcal{M}_{k}, \\ h_{T} & \text { if } T \in \mathcal{T}_{k} \backslash \mathcal{M}_{k} .\end{cases}
$$

Finally, the argument continues as in Lemma 4.1 and this completes the proof.

Lemma 4.3 (Reduction of inconsistency estimator). If $\mu_{3}:=1-\xi^{2} 2^{-\frac{2 b}{d-1}}$, then the following estimate holds:

$$
\rho_{k+1}^{2} \leq \rho_{k}^{2}-\mu_{3} \rho_{k}^{2}\left(\mathcal{M}_{k}\right) .
$$

Proof. The proof is similar to that of Lemma 4.1 and is thus omitted.

Since the finite element spaces $\mathbb{V}_{k}$ are no longer nested, the usual orthogonality property fails [11, 16, 17, 18. Orthogonality is replaced by quasi-orthogonality, very much in the spirit of [15].

Lemma 4.4 (Quasi-orthogonality). If $Q=8\|q\|_{L^{\infty}(\Omega)}^{3}$ as in Lemma 2.4, then

$$
e_{k+1}^{2} \leq e_{k}^{2}-\frac{1}{2} E_{k}^{2}+2 Q^{2} \zeta_{k+1}^{2} .
$$


Proof. We first set the bilinear forms

$$
\mathcal{B}(u, v):=\int_{\Gamma} \nabla_{\Gamma} u \nabla_{\Gamma}^{T} v \quad \text { and } \quad \mathcal{B}_{k}(u, v):=\int_{\Gamma_{k}} \nabla_{\Gamma_{k}} u \nabla_{\Gamma_{k}}^{T} v .
$$

By symmetry of $\mathcal{B}$, we have

$$
e_{k+1}^{2}=e_{k}^{2}-E_{k}^{2}-2 \mathcal{B}\left(u-U_{k+1}, U_{k+1}-U_{k}\right) .
$$

Since $V:=U_{k+1}-U_{k} \in \mathbb{\mathbb { V }}_{k+1}$, we have

$$
\mathcal{B}\left(u-U_{k+1}, U_{k+1}-U_{k}\right)=\mathcal{B}(u, V)-\mathcal{B}\left(U_{k+1}, V\right)=\int_{\Gamma} f V-\mathcal{B}\left(U_{k+1}, V\right) .
$$

Invoking (3.3) and (1.9), we can write $\int_{\Gamma} f V=\mathcal{B}_{k+1}\left(U_{k+1}, V\right)$. Therefore, (2.8) implies

$$
\mathcal{B}\left(u-U_{k+1}, U_{k+1}-U_{k}\right)=\mathcal{B}_{k+1}\left(U_{k+1}, V\right)-\mathcal{B}\left(U_{k+1}, V\right)=\int_{\Gamma} \nabla_{\Gamma} U_{k+1} \mathbf{E}_{k+1} \nabla_{\Gamma}^{T} V .
$$

In view of (1.14), (1.15), and (2.12), we see that

$$
\left\|\nabla_{\Gamma} U_{k+1} \mathbf{E}_{k+1}\right\|_{L^{2}(\Gamma)} \leq Q\left(\sum_{T \in \mathcal{T}_{k+1}}\left\|\nu-\nu_{k+1}\right\|_{L^{\infty}(\widehat{T})}^{2}\left\|\nabla_{\Gamma} U_{k+1}\right\|_{L^{2}(\widetilde{T})}^{2}\right)^{1 / 2}=Q \zeta_{k+1}
$$

and thus

$$
-2 \mathcal{B}\left(u-U_{k+1}, V\right) \leq 2 Q^{2} \zeta_{k+1}^{2}+\frac{1}{2} E_{k}^{2} .
$$

This shows (4.7), as desired.

4.2. Conditional contraction property of AFEM. We are now in a position to prove the main result of this paper.

Theorem 4.1 (Conditional contraction property of AFEM). If $\left\|q\left(\nu-\nu_{k}\right)\right\|_{L^{\infty}(\Gamma)} \leq$ $1 / 2$ for all $k \geq 0$, then there exist positive constants $\gamma_{0}, \beta_{1}, \beta_{2}, \Lambda$ and $\alpha<1$, depending on the shape regularity of $\mathcal{T}_{0}(\Omega)$, the Lipschitz constant $\|\nabla z\|_{L^{\infty}(\Omega)}$ of the function $z$ defining $\Gamma$, the polynomial degree $n \geq 1$, and the parameters $0<\theta, \xi<1$ of AFEM so that if $\mathcal{E}_{k}$ is the total error estimator defined in MARK, namely,

$$
\mathcal{E}_{k}^{2}=\eta_{k}^{2}+\beta_{1} \zeta_{k}^{2}+\beta_{2} \rho_{k}^{2}
$$

and $\lambda_{k} \leq \Lambda$, then

$$
e_{k+1}^{2}+\gamma_{0} \mathcal{E}_{k+1}^{2} \leq \alpha\left(e_{k}^{2}+\gamma_{0} \mathcal{E}_{k}^{2}\right) .
$$

Proof. Let $\gamma_{0}, \gamma_{1}, \gamma_{2}>0$ be parameters to be determined later and let us define $\mu=\min \left(\mu_{1}, \mu_{2}, \mu_{3}\right)<1$ with $\mu_{1}, \mu_{2}, \mu_{3}$ given in Lemmas 4.1, 4.2 and 4.3. In view of (4.7), (4.1), (4.3), and (4.6) we can write

$$
\begin{aligned}
e_{k+1}^{2} & +\gamma_{0} \eta_{k+1}^{2}+\gamma_{1} \zeta_{k+1}^{2}+\gamma_{2} \rho_{k+1}^{2} \leq e_{k}^{2} \\
& +\left(-\frac{1}{2}+\left[\gamma_{0} C_{2}+\left(\gamma_{1}+2 Q^{2}\right) \lambda_{k}^{2}\right]\left(1+\delta^{-1}\right)\right) E_{k}^{2} \\
& +(1+\delta) \gamma_{0}\left(\eta_{k}^{2}-\mu \eta_{k}^{2}\left(\mathcal{M}_{k}\right)\right) \\
& +(1+\delta) \gamma_{1}\left(\zeta_{k}^{2}-\mu \zeta_{k}^{2}\left(\mathcal{M}_{k}\right)\right)+\left(\gamma_{0}\left(1+\delta^{-1}\right) C_{2}+(1+\delta) 2 Q^{2}\right) \zeta_{k}^{2} \\
& +(1+\delta) \gamma_{2}\left(\rho_{k}^{2}-\mu \rho_{k}^{2}\left(\mathcal{M}_{k}\right)\right)+\gamma_{0}\left(1+\delta^{-1}\right) C_{2} \rho_{k}^{2}
\end{aligned}
$$


Regrouping terms of the third, fourth, and fifth rows containing the factor $\mu$, and using (3.6), we obtain

$$
\begin{aligned}
& \gamma_{0} \eta_{k}^{2}\left(\mathcal{M}_{k}\right)+\gamma_{1} \zeta_{k}^{2}\left(\mathcal{M}_{k}\right)+\gamma_{2} \rho_{k}^{2}\left(\mathcal{M}_{k}\right) \\
& \quad=\gamma_{0}\left(\eta_{k}^{2}\left(\mathcal{M}_{k}\right)+\beta_{1} \zeta_{k}^{2}\left(\mathcal{M}_{k}\right)+\beta_{2} \rho_{k}^{2}\left(\mathcal{M}_{k}\right)\right) \\
& \quad \geq \gamma_{0} \theta^{2}\left(\eta_{k}^{2}+\beta_{1} \zeta_{k}^{2}+\beta_{2} \rho_{k}^{2}\right)
\end{aligned}
$$

provided

$$
\beta_{1}=\frac{\gamma_{1}}{\gamma_{0}}, \quad \beta_{2}=\frac{\gamma_{2}}{\gamma_{0}} .
$$

We split the term $\eta_{k}^{2}$ into two equal pieces and replace one by the upper bound from Lemma 3.1

$$
\eta_{k}^{2} \geq C_{1}^{-1} e_{k}^{2}-\zeta_{k}^{2}
$$

Therefore,

$$
\begin{aligned}
e_{k+1}^{2}+ & \gamma_{0} \eta_{k+1}^{2}+\gamma_{1} \zeta_{k+1}^{2}+\gamma_{2} \rho_{k+1}^{2} \leq\left(1-\frac{\mu \gamma_{0}(1+\delta) \theta^{2}}{2 C_{1}}\right) e_{k}^{2} \\
& +\gamma_{0}(1+\delta)\left(1-\frac{\mu \theta^{2}}{2}\right) \eta_{k}^{2} \\
& +\gamma_{1}\left((1+\delta)\left(1-\mu \theta^{2}\right)+(1+\delta) \frac{2 Q^{2}}{\gamma_{1}}+\frac{\gamma_{0}}{\gamma_{1}}\left(1+\delta^{-1}\right) C_{2}\left(1+\frac{\delta \mu \theta^{2}}{2 C_{2}}\right)\right) \zeta_{k}^{2} \\
& +\gamma_{2}\left((1+\delta)\left(1-\mu \theta^{2}\right)+\frac{\gamma_{0}}{\gamma_{2}}\left(1+\delta^{-1}\right) C_{2}\right) \rho_{k}^{2} \\
& +\left(-\frac{1}{2}+\gamma_{0}\left(1+\delta^{-1}\right) C_{2}+\left(\gamma_{1}+2 Q^{2}\right)\left(1+\delta^{-1}\right) \lambda_{k}^{2}\right) E_{k}^{2}
\end{aligned}
$$

We now choose the parameters $\gamma_{0}, \gamma_{1}, \gamma_{2}$, and $\delta$. We start with $\delta$ being chosen sufficiently small so that

$$
(1+\delta)\left(1-\frac{\mu \theta^{2}}{2}\right)=1-\frac{\mu \theta^{2}}{4}=\alpha_{1}<1 .
$$

We next choose $\gamma_{0}$ in such a way that

$$
\frac{1}{4}=\gamma_{0}\left(1+\delta^{-1}\right) C_{2} \quad \Rightarrow \quad \gamma_{0}(1+\delta)=\frac{\delta}{4 C_{2}} .
$$

Since, as $\gamma_{1} \uparrow \infty$,

$$
(1+\delta)\left(1-\mu \theta^{2}\right)+\frac{(1+\delta) 2 Q^{2}}{\gamma_{1}}+\underbrace{\frac{\gamma_{0}\left(1+\delta^{-1}\right) C_{2}}{\gamma_{1}}}_{=1 /\left(4 \gamma_{1}\right)}\left(1+\frac{\delta \mu \theta^{2}}{2 C_{2}}\right) \longrightarrow(1+\delta)\left(1-\mu \theta^{2}\right),
$$

we select $\gamma_{1}>0$ so that

$$
(1+\delta)\left(1-\mu \theta^{2}\right)+\frac{(1+\delta) 2 Q^{2}}{\gamma_{1}}+\frac{1}{4 \gamma_{1}}\left(1+\frac{\delta \mu \theta^{2}}{2 C_{2}}\right)=1-\frac{\mu \theta^{2}}{4}=\alpha_{1} ;
$$

hence the prefactor of $\zeta_{k}^{2}$ becomes $\alpha_{1} \gamma_{1}$. Likewise, we pick the constant $\gamma_{2}>0$ so that

$$
\underbrace{(1+\delta)\left(1-\mu \theta^{2}\right)}_{<1-\frac{\mu \theta^{2}}{4}}+\underbrace{\frac{\gamma_{0}\left(1+\delta^{-1}\right) C_{2}}{\gamma_{2}}}_{=1 /\left(4 \gamma_{2}\right)}=1-\frac{\mu \theta^{2}}{4}=\alpha_{1},
$$


whence the prefactor of $\rho_{k}^{2}$ is $\alpha_{1} \gamma_{2}$. Finally, the multiplier of $e_{k}$ becomes

$$
1-\frac{\mu \delta \theta^{2}}{8 C_{1} C_{2}}=\alpha_{0}<1
$$

Now, let $\alpha:=\max \left(\alpha_{0}, \alpha_{1}\right)<1$ and the threshold

$$
\Lambda:=\frac{1}{2 \sqrt{\left(\gamma_{1}+2 Q^{2}\right)\left(1+\delta^{-1}\right)}} .
$$

Then we find the relation

$$
e_{k+1}^{2}+\underbrace{\gamma_{0} \eta_{k+1}^{2}+\gamma_{1} \zeta_{k+1}^{2}+\gamma_{2} \rho_{k+1}^{2}}_{=\gamma_{0} \mathcal{E}_{k+1}^{2}} \leq \alpha(e_{k}^{2}+\underbrace{\gamma_{0} \eta_{k}^{2}+\gamma_{1} \zeta_{k}^{2}+\gamma_{2} \rho_{k}^{2}}_{=\gamma_{0} \mathcal{E}_{k}^{2}})+\frac{1}{4}\left(-1+\frac{\lambda_{k}^{2}}{\Lambda^{2}}\right) E_{k}^{2}
$$

and conclude that for $\lambda_{k} \leq \Lambda$ we obtain a contraction, as asserted.

Remark 4.1 (Simpler proof). This proof does not use the a posteriori lower bound nor the interior node property of Morin et al. [16, 17, 18. This is quite convenient for implementation, especially for $d \geq 4$. The analysis is in the spirit of Cascón et al. 4, but a few new ingredients are added to account for geometry.

Remark 4.2 (Polynomial degree). This proof is valid for any polynomial degree $n \geq 1$, which does not enter explicitly because we avoid using a lower bound.

\section{NUMERICAL EXPERIMENT}

To illustrate our main result, we present a numerical experiment based on the AFEM described above. The algorithm was implemented within the finite element toolbox ALBERTA developed by Schmidt and Siebert [20], and the graphics were produced with GMV [19].

We use our AFEM to compute approximate solutions of the Laplace-Beltrami operator on $\Gamma$ with homogeneous Dirichlet boundary conditions, where $\Gamma$ is the piecewise smooth graph of

$$
z(x, y)= \begin{cases}0.25 \sin (2 \pi x) \sin (4 \pi y), & \text { if } x y>0, \\ 0, & \text { otherwise }\end{cases}
$$

over the region $\Omega:=(-0.5,1.5) \times(-1.5,0.5) \backslash[0.5,1,5) \times(-1.5,-0.5]$, i.e., the usual L-shaped domain shifted 0.5 to the right and 0.5 downward. The surface $\Gamma$ has a variable curvature and exhibits jumps of the normal across the coordinate axes (see Figure 4, top row); $\Gamma$ is Lipschitz.

The exact solution $u$ is unknown to us but satisfies the boundary value problem:

$$
\left\{\begin{aligned}
-\Delta_{\Gamma} u=f & \text { in } \Gamma, \\
u=0 & \text { on } \partial \Gamma, \quad \text { with } \quad f(x, y)=1-5 e^{-50\left[(x-0.5)^{2}+(y+0.05)^{2}\right]} .
\end{aligned}\right.
$$

We thus expect $u$ to exhibit a singularity at the re-entrant corner, plus some interesting behavior around the (negative) peak of $f$, namely at $(0.5,-0.05)$, along with large variations (in $\Omega$ ) where the surface oscillates, especially near parts of $\partial \Omega$.

We implemented AFEM with polynomial degree $n=1$ and parameters:

$$
\theta=0.5, \quad \beta_{1}=1, \quad \beta_{2}=1, \quad \text { and } \quad \xi=0.9 \text {. }
$$

The number of bisections $b$ performed to marked elements was set to $b=2$, which is customary for two-dimensional meshes. The constant $C_{1}$ of the upper bound (3.4) was set to one which, in contrast to the other parameters in (5.1), has no effect in 
the behavior of AFEM; $C_{1}$ is important to stop AFEM reliably. Even though our theory guarantees a contraction for certain values of parameters $\beta_{1}$ and $\beta_{2}$, we found that the simple choice made here leads to linear convergence and a quasi-optimal behavior of AFEM, as described below.

\begin{tabular}{|c|c|c|c|c|c|c|c|c|}
\hline \multirow{2}{*}{$\begin{array}{c}\text { Iter. } \\
k\end{array}$} & \multirow{2}{*}{$\begin{array}{c}\text { No. of } \\
\text { elements }\end{array}$} & \multicolumn{2}{|c|}{ Marked elements } & \multicolumn{5}{|c|}{ Estimators } \\
\hline & & (3.6) & (3.7)-(3.8) & $\eta_{k}$ & $\lambda_{k}$ & $\rho_{k}$ & $\zeta_{k}$ & $\left(\eta_{k}^{2}+\zeta_{k}^{2}\right)^{1 / 2}$ \\
\hline 0 & 24 & 12 & 60 & 1.621 & 1.180 & 0.449 & 0.7214 & 1.682 \\
\hline 1 & 280 & 15 & 0 & 0.716 & 1.056 & 0.459 & 0.2868 & 0.850 \\
\hline 2 & 364 & 37 & 0 & 0.633 & 1.056 & 0.417 & 0.2713 & 0.758 \\
\hline 3 & 555 & 27 & 0 & 0.546 & 1.056 & 0.304 & 0.2093 & 0.625 \\
\hline 4 & 714 & 38 & 0 & 0.494 & 1.056 & 0.265 & 0.1817 & 0.560 \\
\hline 5 & 940 & 51 & 0 & 0.433 & 1.056 & 0.242 & 0.0985 & 0.496 \\
\hline 6 & 1244 & 78 & 0 & 0.387 & 0.974 & 0.187 & 0.0741 & 0.430 \\
\hline 7 & 1721 & 117 & 0 & 0.333 & 0.816 & 0.165 & 0.0612 & 0.372 \\
\hline 8 & 2427 & 184 & 16 & 0.285 & 0.816 & 0.139 & 0.0474 & 0.317 \\
\hline 9 & 3498 & 334 & 10 & 0.241 & 0.816 & 0.114 & 0.0325 & 0.266 \\
\hline 10 & 5339 & 312 & 29 & 0.196 & 0.816 & 0.091 & 0.0215 & 0.216 \\
\hline 11 & 7237 & 486 & 21 & 0.169 & 0.816 & 0.078 & 0.0159 & 0.186 \\
\hline 12 & 10118 & 611 & 55 & 0.143 & 0.816 & 0.067 & 0.0116 & 0.158 \\
\hline 13 & 13687 & 1030 & 55 & 0.124 & 0.544 & 0.058 & 0.0089 & 0.137 \\
\hline 14 & 19441 & 1391 & 201 & 0.105 & 0.544 & 0.048 & 0.0068 & 0.115 \\
\hline 15 & 28246 & 1590 & 120 & 0.087 & 0.544 & 0.040 & 0.0045 & 0.096 \\
\hline 16 & 37563 & 2305 & 96 & 0.075 & 0.544 & 0.035 & 0.0033 & 0.083 \\
\hline 17 & 49772 & 4546 & 370 & 0.065 & 0.544 & 0.030 & 0.0025 & 0.072 \\
\hline 18 & 74457 & 4595 & 398 & 0.054 & 0.303 & 0.024 & 0.0018 & 0.059 \\
\hline 19 & 100037 & 6510 & 881 & 0.046 & 0.303 & 0.021 & 0.0013 & 0.051 \\
\hline 20 & 139461 & 10946 & 736 & 0.039 & 0.303 & 0.018 & 0.0009 & 0.043 \\
\hline
\end{tabular}

TABLE 1. Numerical information on the behavior of AFEM. Iteration number (column 1), number of elements (column 2), number of elements marked for refinement (columns 3-4), and error estimators (columns 5-9). Column 3 displays the number of elements marked in order to fulfill Dörfler's condition (3.6), and the fourth column displays the total number of additional elements marked (in an inner loop) in order to fulfill (3.7) and (3.8). The last column displays the value of the upper error bound (3.4) in Lemma 3.1 with $C_{1}=1$, which decreases as $N^{-1 / 2}$, with $N$ being the number of elements. The decay of the various estimators can be seen in Figure 3

In Table1 we present all the numerical information about the behavior of AFEM. The first and second column indicate the iteration number and the number of elements of the current mesh, respectively. The third column displays the number of elements marked in order to fulfill Dörfler's condition (3.6), whereas the fourth column displays the total number of additional elements marked (in an inner loop) in order to fulfill (3.7) and (3.8). This number is high in the first iteration due to bad surface resolution, but it is then zero for seven consecutive iterations. Afterwards the number of elements marked to fulfill (3.7)-(3.8) stays around eight percent of the number of elements marked to fulfill (3.6). Another run of AFEM with $\xi=0.95$ instead of $\xi=0.9$, but not reported here, marked 60 elements in the first iteration (in order to ensure (3.7)-(3.8)), and zero in all the subsequent iterations; this is consistent with Remark 3.2. Finally, the last five columns of Table 1 display the 


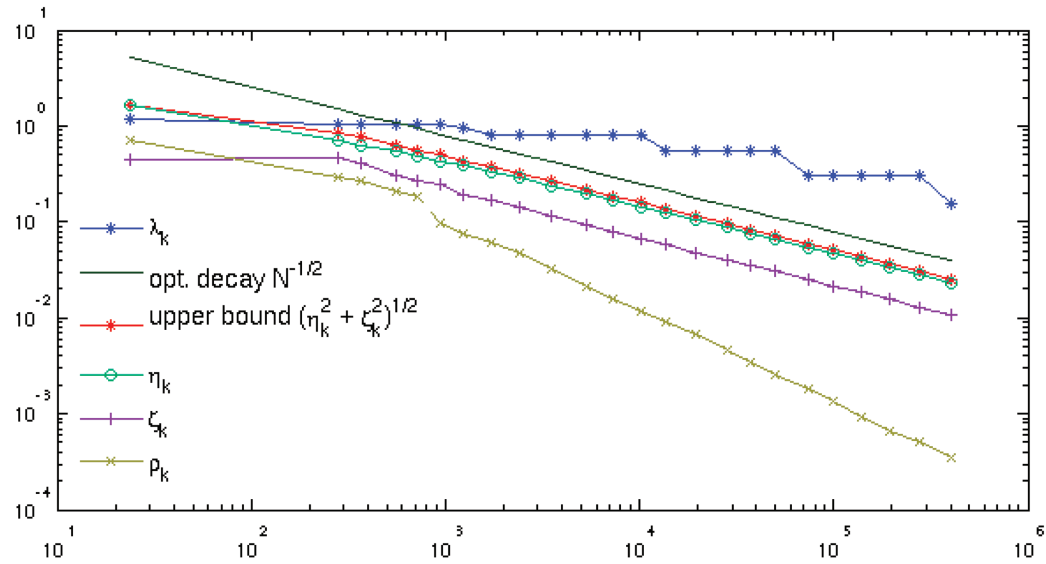

FIGURE 3. Logarithmic plot of estimators versus number $N$ of elements. All of them, except for $\lambda_{k}$, decrease at the expected optimal rate $N^{-1 / 2}$ for linear finite elements. In particular, the upper error bound $\left(\eta_{k}^{2}+\zeta_{k}^{2}\right)^{1 / 2}$ of Lemma 3.1 with $C_{1}=1$ (last column of Table 1) exhibits optimal decay with respect to the number of elements, indicating the same behavior for the exact energy error $\left\|\nabla_{\Gamma}\left(u-u_{k}\right)\right\|_{L^{2}(\Gamma)}$.

a posteriori error estimators that guide AFEM, namely, the energy error estimator $\eta_{k}$, the surface estimator $\lambda_{k}$, the term $\rho_{k}$ measuring the lack of consistency of the right-hand side, and the geometric estimator $\zeta_{k}$. The last column displays the upper error bound (3.4) in Lemma 3.1 with $C_{1}=1$.

Figure 3 depicts the behavior of all the a posteriori error estimators introduced in the manuscript. All of them, except for $\lambda_{k}$, decrease at the expected optimal rate $N^{-1 / 2}$ for linear finite elements. In particular, we can see that the upper error bound $\left(\eta_{k}^{2}+\zeta_{k}^{2}\right)^{1 / 2}$ of Lemma 3.1 with $C_{1}=1$ (last column of Table [1) decays optimally with respect to the number of elements, indicating that the same happens with the exact error, which is unknown to us. The term $\rho_{k}$ measures the lack of consistency of the right-hand side with the discrete surface and decreases faster than the other quantities, allowing us to conclude that it has a minimal effect on the behavior of the algorithm. The quantity $\lambda_{k}$ measures surface resolution, regardless of the solution being computed, and exhibits a suboptimal decay. It turns out, however, that this is not essential for the optimal decay of the total error estimator $\mathcal{E}_{k}$ because the only (theoretical) requirement for $\lambda_{k}$ is that it is locally monotonically decreasing - which is guaranteed by AFEM - and also that it is below the threshold $\Lambda$. This threshold is unknown but plays no role in the decision making of AFEM. However, $\Lambda$ could be estimated from the proof of Theorem 4.1, if desired. The reported values of $\lambda_{k}$ are relatively large and might not satisfy $\lambda_{k} \leq \Lambda$ for the theory to apply (see Theorem 4.1), thereby suggesting that the theory is a bit pessimistic and cannot fully explain the optimal behavior of AFEM.

Finally, in Figure 4 we show three meshes at iteration steps $k=6,9,12$, as viewed from the point $(1,-1,1)$ in space (first row), and also from the top (second row). These meshes reflect the relatively large variation of the curvature of $\Gamma$ near the pieces $\{-0.5\} \times[-1.5,0]$ and $[0,1.5] \times\{0.5\}$ of $\partial \Omega$ as well as the re-entrant corner, but not quite the steep behavior of the forcing function $f$ at $(0.5,-0.05)$. 
We also observe that the meshes in the second quadrant are about uniform because both $\Gamma$ is flat and the solution is smooth. The jump singularities of $\Gamma$ across the cordinate axes do not yield further refinement because they are exactly matched by the initial mesh. In the third row of Figure 4 we depict the contour lines of the discrete solutions in order to reveal their behavior.
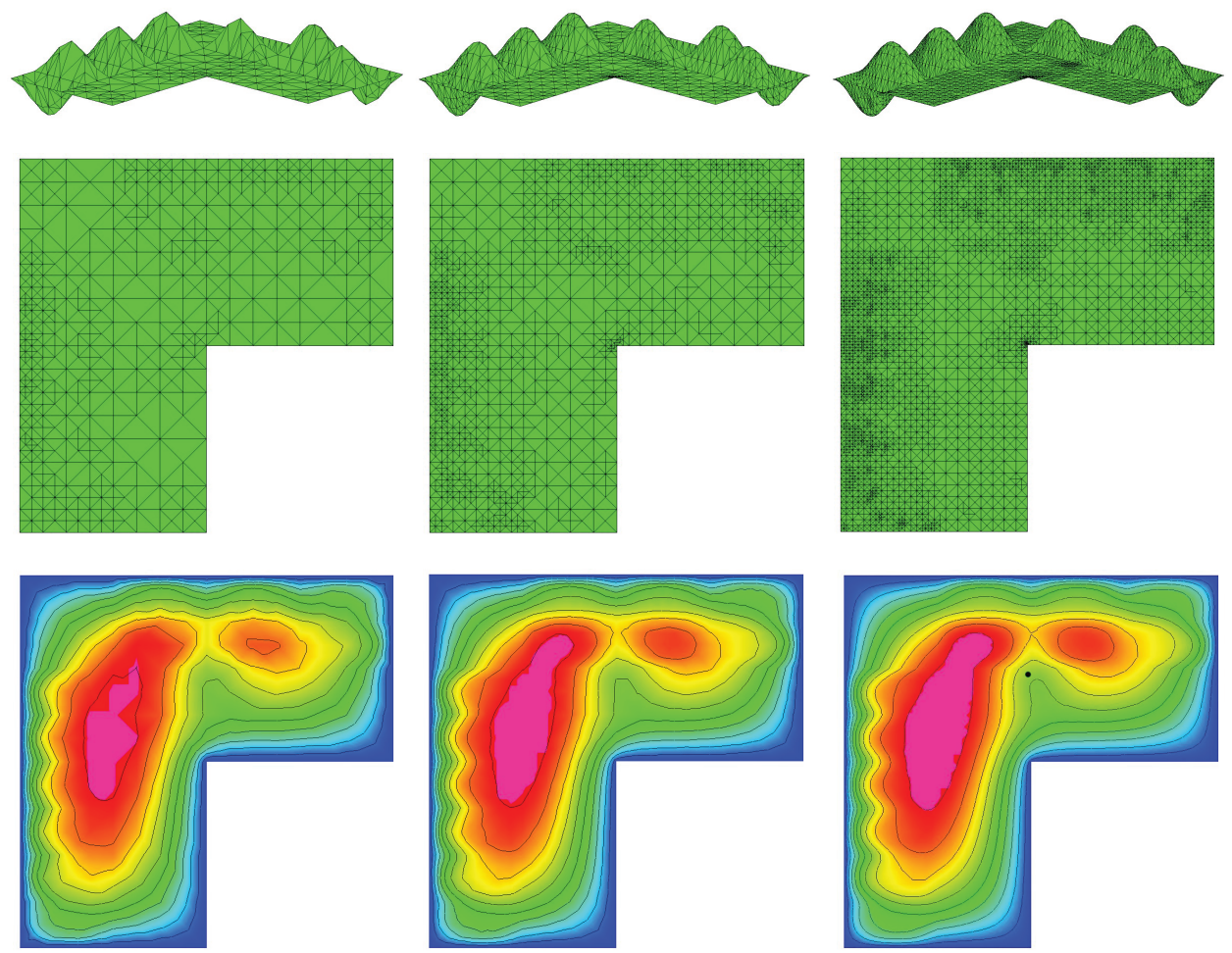

FIGURE 4. Meshes and discrete solutions after 6, 9, and 12 iterations of AFEM, respectively. The meshes show that mesh refinement is driven by resolution of both the corner singularity at the origin as well as the curvature of the surface, whereas the peak of the source term $f$ plays a secondary role. The last row depicts the contour lines of the corresponding discrete solutions, viewed from top, which show a mild effect of $f$ around its peak, indicated with a dot in the third bottom figure.

\section{REFERENCES}

1. M. Ainsworth and J. T. Oden, A Posteriori Error Estimation in Finite Element Analysis, John Wiley \& Sons, Inc., 2000. MR.1885308 (2003b:65001)

2. E. Bänsch, P. Morin, and R. H. Nochetto, Surface diffusion of graphs: Variational formulation, error analysis and simulation, SIAM J. Numer. Anal., 42, 2 (2004), 773-799. MR2084235 (2005h:65160)

3. S. Brenner and R. Scott, The Mathematical Theory of Finite Element Methods, Springer Texts in Applied Mathematics 15, 2008. MR2373954 (2008m:65001)

4. J. M. Cascón, Ch. Kreuzer, R. H. Nochetto, and K. Siebert, Quasi-optimal convergence rate for an adaptive finite element method, SIAM J. Numer. Anal. 46 (2008), 2524-2550. MR 2421046 (2009h:65174) 
5. J. M. Cascón, K. Mekchay, P. Morin and R. H. Nochetto, Quasi-optimal convergence rate for AFEM for the Laplace-Beltrami operator on parametric surfaces, (in preparation).

6. Z. Chen and J. Feng, An adaptive finite element algorithm with reliable and efficient error control for linear parabolic problems, Math. Comp. 73, (2006), 1167-1042. MR2047083 (2005e:65131)

7. P. G. Ciarlet, The Finite Element Method for Elliptic Problems, North-Holland, Amsterdam, 1978; reprinted, Classics Appl. Math. 40, SIAM, Philadelphia, 2002. MR0520174 (58:25001)

8. P. Clément, Approximation by finite element functions using local regularizations, RAIRO Modél. Math. Anal. Numér. 2 (1975), 77-84. MR0400739 (53:4569)

9. A. Demlow, Higher-order finite element methods and pointwise error estimates for elliptic problems on surfaces, SIAM J. Numer. Anal. 47 (2009), 805-827. MR2485433 (2010a:65233)

10. A. Demlow and G. Dziuk, An adaptive finite element method for the Laplace-Beltrami operator on implicitly defined surfaces, SIAM J. Numer. Anal. 45 (2007), 421-442. MR2285862 (2008c:65320)

11. W. Dörfler, A convergent adaptive algorithm for Poisson's equation, SIAM J. Numer. Anal. 33 (1996), 1106-1124. MR.1393904 (97e:65139)

12. W. Dörfler and M. Rumpf, An adaptive strategy for elliptic problems including a posteriori controlled boundary approximation, Math. Comp. 67 (1998), 1361-1382. MR1489969 (99b:65141)

13. G. Dziuk, Finite elements for the Beltrami operator on arbitrary surfaces, Partial Differential Equations and Calculus of Variations, Lecture Notes in Mathematics 1357, Springer, Berlin, Heidelberg, New York, London, Paris, Tokyo, 1988, 142-155. MR976234 (90i:65194)

14. K. Mekchay, Convergence of adaptive finite element methods, Ph.D. Dissertation, University of Maryland, December 2005.

15. K. Mekchay and R. H. Nochetto, Convergence of adaptive finite element methods for general second order linear elliptic PDE, SIAM J. Numer Anal. 43 (2005), 1803-1827. MR2192319 (2006i:65201)

16. P. Morin, R. H. Nochetto, and K. G. Siebert, Data oscillation and convergence of adaptive FEM, SIAM J. Numer. Anal. 38 (2000), 466-488. MR.1770058 (2001g:65157)

17. P. Morin, R. H. Nochetto, and K. G. Siebert, Convergence of adaptive finite element methods, SIAM Review 44 (2002), 631-658. MR.1980447

18. P. Morin, R. H. Nochetto, and K. G. Siebert, Local problems on stars: a posteriori error estimation, convergence and performance, Math. Comp. 72 (2003), 1067-1097. MR1972728 (2004d:65129)

19. F. A. Ortega, GMV: General Mesh Viewer users Manual, Version 1.8. Los Alamos National Laboratory, LAUR 95-2986, 1995.

20. A. Schmidt and K. G. Siebert, Design of Adaptive Finite Element Software. The Finite Element Toolbox ALBERTA, Lecture Notes in Computational Science and Engineering 42, Springer, Berlin, 2005. MR 2127659 (2005i:65003)

21. R. Verfürth, A Review of A Posteriori Error Estimation and Adaptive Mesh-Refinement Technique, Wiley-Teubner, Chichester, 1996.

Department of Mathematics, Faculty of Science, Chulalongkorn University, Phyathai, Bangkok 10330, Thailand - And - University of Maryland, College Park, MaryLAND 20742

E-mail address: k.mekchay@gmail.com

Instituto de Matemática Aplicada del Litoral, Universidad Nacional del Litoral, CONICET, Güemes 3450, S3000GLN Santa Fe, Argentina

E-mail address: pmorin@santafe-conicet.gov.ar

Department of Mathematics and Institute for Physical Science and Technology, University of Maryland, College Park, Maryland 20742

E-mail address: rhn@math.umd.edu 